\title{
$\beta$-Glycosides of Hydroxyproline via an Umpolung Approach
}

\author{
Karl J. Shaffer and Carol M. Taylor* \\ Institute of Fundamental Sciences, Massey University, Private Bag \\ 11-222, Palmerston North, New Zealand.
}

C.M.Taylor@massey.ac.nz

Received Date (will be automatically inserted after manuscript is accepted)

\section{SUPPORTING INFORMATION}

Experimental procedures for the preparation of compounds and their ${ }^{1} \mathrm{H}$ and ${ }^{13} \mathrm{C}$ NMR spectra. Compounds are listed in the order that they appear in the Letter:

\begin{tabular}{|l|c|c|c|c|}
\hline Scheme & Compound & Procedure & ${ }^{\mathbf{1}}$ H NMR & ${ }^{\mathbf{1 3}} \mathbf{C}$ NMR \\
\hline Scheme 4 & $\mathbf{7}$ & $\mathrm{S} 3$ & $\mathrm{~S} 13$ & $\mathrm{~S} 14$ \\
\hline Scheme 5 & trans-8 & $\mathrm{S} 3$ & $\mathrm{~S} 15$ & $\mathrm{~S} 16$ \\
\cline { 2 - 5 } & trans-5 & $\mathrm{S} 4$ & $\mathrm{~S} 17$ & $\mathrm{~S} 18$ \\
\cline { 2 - 5 } & cis-9 & $\mathrm{S} 5$ & $\mathrm{~S} 19$ & $\mathrm{~S} 20$ \\
\cline { 2 - 5 } & cis-8 & $\mathrm{S} 5$ & $\mathrm{~S} 21$ & $\mathrm{~S} 22$ \\
\cline { 2 - 5 } & cis-5 & $\mathrm{S} 6$ & $\mathrm{~S} 23$ & $\mathrm{~S} 24$ \\
\hline Table 1 & trans-4 & $\mathrm{S} 6$ & $\mathrm{~S} 25$ & $\mathrm{~S} 26$ \\
\hline \multirow{3}{*}{ Scheme 6 } & cis-4 & $\mathrm{S} 7$ & $\mathrm{~S} 27$ & $\mathrm{~S} 28$ \\
\cline { 2 - 5 } & trans-14 & $\mathrm{S} 8$ & $\mathrm{~S} 29$ & $\mathrm{~S} 30$ \\
\cline { 2 - 5 } & cis-14 & $\mathrm{S} 9$ & $\mathrm{~S} 31$ & $\mathrm{~S} 32$ \\
\cline { 2 - 5 } & $\mathbf{1 5}$ & $\mathrm{S} 9$ & $\mathrm{~S} 33$ & $\mathrm{~S} 34$ \\
\cline { 2 - 5 } & $\mathbf{1 6}$ & $\mathrm{S} 10$ & $\mathrm{~S} 35$ & $\mathrm{~S} 36$ \\
\cline { 2 - 5 } & $\mathbf{1 7}$ & $\mathrm{S} 11$ & $\mathrm{~S} 37$ & $\mathrm{~S} 38$ \\
\cline { 2 - 5 } & $\mathbf{1 8}$ & $\mathrm{S} 11$ & $\mathrm{~S} 39$ & $\mathrm{~S} 40$ \\
\hline
\end{tabular}




\section{General Experimental Methods}

Reagents were used as received from commercial suppliers, with the following exceptions. Dichloromethane was freshly distilled from calcium hydride. Dimethylformamide was distilled from barium oxide and stored over $4 \AA$ molecular sieves. Methanol was distilled from magnesium turnings and iodine and stored over $4 \AA$ molecular sieves. Pyridine was distilled from $\mathrm{CaH}_{2}$ and stored over $\mathrm{KOH}$ pellets. Tetrahydrofuran was freshly distilled from sodiumbenzophenone. Toluene was freshly distilled from sodium. Molecular sieves were powdered, with $4 \AA$ pores, and were flame-dried immediately prior to use.

Potassium benzoate was prepared by mixing benzoic acid (2.000 g, $16.39 \mathrm{mmol} 2.0$ equiv.) and potassium carbonate $(1.132 \mathrm{~g}, 8.19 \mathrm{mmol}, 1.0$ equiv. $)$ in water $(50 \mathrm{~mL})$. The solution was warmed for $30 \mathrm{~min}$ then the solvent was removed and the resulting white powder was azeotroped twice with toluene. The mass of solid obtained was consistent with the formation of potassium benzoate and the $\mathrm{pH}$ was neutral.

Reactions were followed by TLC on aluminium-backed silica gel $60 \mathrm{~F}_{254}$ sheets from E-Merck, visualized under UV light or by staining with anisaldehyde. Column chromatography was performed using Scharlau silica gel 60, 0.04-0.06 mm, 230-400 mesh.

NMR spectra were recorded on a Bruker Avance 500 spectrometer, operating at $500 \mathrm{MHz}\left({ }^{1} \mathrm{H}\right)$ or $125 \mathrm{MHz}\left({ }^{13} \mathrm{C}\right)$. Chemical shifts are reported relative to TMS $\left({ }^{1} \mathrm{H}\right)$ or residual solvent $\left({ }^{13} \mathrm{C}\right)$ as an internal reference. Full NMR assignments were made using ${ }^{1} \mathrm{H},{ }^{13} \mathrm{C}, \mathrm{COSY}$ and HMQC experiments. Fmoc-Pro derivatives invariably exist as mixture of rotamers about the carbamate $\mathrm{C}-\mathrm{N}$ bond, typically in a 1:1 ratio. Thus, an integral of $0.5 \mathrm{H}$ refers to one proton in one of the rotamers.

High resolution mass spectra were recorded on a VG 7070 mass spectrometer, operating at a nominal voltage of $70 \mathrm{eV}$. Triflates cis-5 and trans -5 were not sufficiently stable to survive the transport required and analysis by HRMS. Evidence for the identity of freshly prepared triflates was obtained by LRMS on an inhouse Micromass M@ldi LR spectrometer.

Optical rotations were recorded on a Perkin Elmer polarimeter, model 341. 


\section{3,4,6-Tri-O-benzyl-D-mannopyranose (7)}

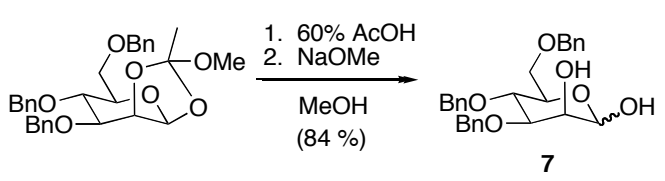

3,4,6-Tri- $O$-benzyl-1,2-(1-methoxyethylidene)- $\beta$-D-mannopyranose ${ }^{1}$ (1.00 g, $1.96 \mathrm{mmol}, 1.00$ equiv.) was suspended in $60 \%$ aq. AcOH $(20 \mathrm{~mL})$ and stirred at RT under $\mathrm{N}_{2}$ for $2 \mathrm{~h}$. A colorless solution formed, which was diluted with EtOAc $(200 \mathrm{~mL})$, washed with water $(2 \times 200 \mathrm{~mL})$ and sat'd aq. $\mathrm{NaHCO}_{3}(200 \mathrm{~mL})$. The organic layer was dried over $\mathrm{MgSO}_{4}$, filtered and concentrated to give a colorless oil $(\sim 1.00 \mathrm{~g})$ which was a mixture of 1-O-acetyl and 2-O-acetyl derivatives. This oil was dissolved in $\mathrm{MeOH}(20 \mathrm{~mL})$ and sodium methoxide (97 mg, $1.79 \mathrm{mmol}, 0.91$ equiv.) was added. The mixture was stirred at RT under $\mathrm{N}_{2}$ overnight. The reaction was neutralised by the addition of Amberlyst IR-120 ( $\left.\mathrm{H}^{+}\right)$, stirred for $30 \mathrm{~min}$, filtered and concentrated. The residue was purified by flash column chromatography, eluting with 1:1 hexanes-EtOAc, to give 7 as a colorless solid that was a mixture of $\alpha$ and $\beta$ anomers $(0.74 \mathrm{~g}, 84 \%) . \quad R_{f} 0.27$ (2:1 EtOAchexanes). NMR data is for the $\alpha$-anomer (major product). ${ }^{1} \mathrm{H} \mathrm{NMR}\left(500 \mathrm{MHz}, \mathrm{CDCl}_{3}\right) \delta 2.77$ (s, 1H, 2-OH), 3.60-3.67 (m, 2H, H6', H6), 3.71 (t, $J=9.4 \mathrm{~Hz}, 1 \mathrm{H}, \mathrm{H} 4), 3.91$ (dd, $J=9.2,3.1 \mathrm{~Hz}$, 1H, H3) 4.98 (s, 1H, H2), 4.02-4.05 (m, 1H, H5), 4.24 (d, 1H, 1-OH), 4.44-4.84 (m, 6H, 3 x $\left.\mathrm{PhCH}_{2} \mathrm{O}\right), 5.24$ (s, 1H, H1), 7.24-7.34 (m, 15H, Ph- $\left.\underline{\mathrm{H}}\right) ;{ }^{13} \mathrm{C}$ NMR (125 MHz, $\left.\mathrm{CDCl}_{3}\right) \delta 68.4(\mathrm{C} 2)$, 69.2 (C6), 70.5 (C5), $71.8\left(\mathrm{PhCH}_{2} \mathrm{O}\right), 73.2\left(\mathrm{PhCH}_{2} \mathrm{O}\right), 74.4$ (C4), $74.9\left(\mathrm{PhCH}_{2} \mathrm{O}\right), 79.6$ (C3), 93.8 (C1), 127.6, 127.6, 127.8, 127.9, 128.0, 128.2, 128.3, 128.4, 137.6, 137.8, 138.1.

\section{$N$-Fluorenylmethoxycarbonyl-trans-4-hydroxy-L-proline allyl ester (trans-8)}

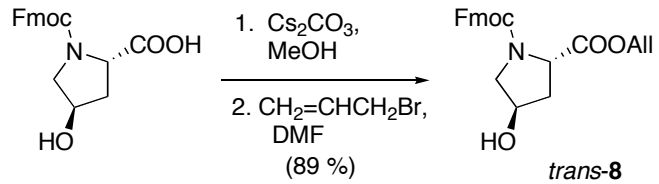

Prepared as described previously ${ }^{2}$ and purified by flash column chromatography, eluting with 1:1 hexanes-EtOAc, to give trans-8 as a colorless oil. $R_{f} 0.40(2: 1$ EtOAc-hexanes $)$. $[\alpha]_{\mathrm{D}}{ }^{20}=-78.5^{\circ}(c$

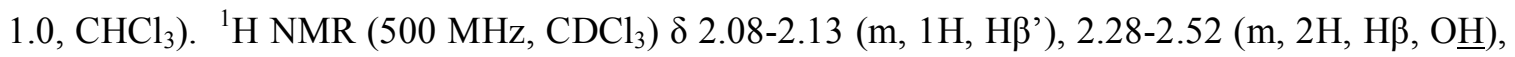
3.55 (d, $J=11.4 \mathrm{~Hz}, 0.5 \mathrm{H}, \mathrm{H} \delta$ '), 3.66 (d, $J=3.0 \mathrm{~Hz}, 1 \mathrm{H}, \mathrm{H} \delta), 3.73$ (dd, $J=11.3,4.4 \mathrm{~Hz}, 0.5 \mathrm{H}$, $\left.\mathrm{H} \delta^{\prime}\right), 4.15 \& 4.26$ (t, $\left.J=6.5 \mathrm{~Hz}, 1 \mathrm{H}, \mathrm{Fmoc}-\mathrm{CH}\right), 4.29-4.65$ (m, 6H, $\underline{\mathrm{H}}_{2} \mathrm{CH}=\mathrm{CH}_{2}$, Fmoc- $\mathrm{CH}_{2}, \mathrm{H} \gamma$, $\mathrm{H} \alpha), 5.18\left(\mathrm{~d}, J=10.5,0.5 \mathrm{H}, \mathrm{CH}=\underline{\mathrm{CH}}_{2} c i s\right), 5.22\left(\mathrm{~d}, J=10.5,0.5 \mathrm{H}, \mathrm{CH}=\mathrm{CH}_{2} c i s\right), 5.26(\mathrm{~d}, J=$

\footnotetext{
${ }^{1}$ Prepared as described previously in detail in the Supporting Information of: Beignet, J.;

Tiernan, J.; Woo, C. H.; Kariukui, B. M.; Cox, L. R. J. Org. Chem. 2004, 69, 6341-6356.

${ }^{2}$ Taylor, C. M.; Weir, C. M. Aust. J. Chem. 2002, 55, 135-140.
} 
$17.8,0.5 \mathrm{H}, \mathrm{CH}=\underline{\mathrm{C}}_{2}$ trans $), 5.32\left(\mathrm{~d}, J=17.8,0.5 \mathrm{H}, \mathrm{CH}=\underline{\mathrm{C}}_{2}\right.$ trans $), 5.79-5.94(\mathrm{~m}, 1 \mathrm{H}$, $\left.\mathrm{C} \underline{\mathrm{H}}=\mathrm{CH}_{2}\right), 7.27-7.30(\mathrm{~m}, 2 \mathrm{H}, \mathrm{Ar}-\underline{\mathrm{H}}), 7.36-7.39$ (m, 2H, Ar- $\left.\underline{\mathrm{H}}\right), 7.52-7.59$ (m, 2H, Ar- $\left.\underline{\mathrm{H}}\right), 7.73-$ $7.75(\mathrm{~m}, 2 \mathrm{H}, \mathrm{Ar}-\underline{\mathrm{H}}) ;{ }^{13} \mathrm{C}$ NMR $\left(125 \mathrm{MHz}, \mathrm{CDCl}_{3}\right) \delta 38.4 \& 39.3(\mathrm{C} \beta), 47.1 \& 47.2$ (Fmoc- $\left.\mathrm{CH}\right)$, $54.6 \& 55.3(\mathrm{C} \delta), 57.6 \& 57.9(\mathrm{C} \alpha), 65.6\left(\underline{\mathrm{CH}}_{2} \mathrm{CH}=\mathrm{CH}_{2}\right), 67.5 \& 67.8\left(\mathrm{Fmoc}^{-} \mathrm{CH}_{2}\right), 69.3 \& 70.1(\mathrm{C} \gamma)$, $118.6 \& 118.8\left(\mathrm{CH}=\underline{\mathrm{C}} \mathrm{H}_{2}\right), 119.9,125.0,127.1,127.7,131.5 \& 131.7\left(\underline{\mathrm{CH}}=\mathrm{CH}_{2}\right), 141.3,143.6$, $154.9($ Fmoc $\underline{\mathrm{C}}=\mathrm{O}), 172.2$ ( $\underline{\mathrm{COOR}})$.

\section{$\mathrm{N}$-Fluorenylmethoxycarbonyl-trans-4-trifluoromethylsulfonyloxy-L-proline allyl ester} (trans-5)

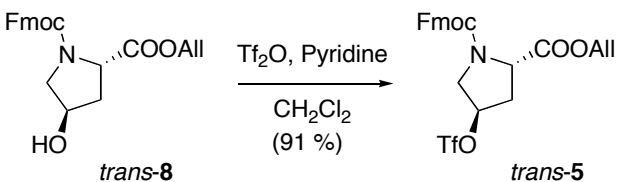

Pyridine ( $87 \mu \mathrm{L}, 84 \mathrm{mg}, 1.07 \mathrm{mmol}, 2.0$ equiv.), followed by triflic anhydride (140 $\mu \mathrm{L}, 226 \mathrm{mg}$, $0.80 \mathrm{mmol}, 1.5$ equiv.), was added to a solution of $N$-fluorenylmethoxycarbonyl-trans-4-hydroxyL-proline allyl ester (trans-8) $\left(210 \mathrm{mg}, 0.53 \mathrm{mmol}, 1.00\right.$ equiv.) in dry $\mathrm{CH}_{2} \mathrm{Cl}_{2}(4 \mathrm{~mL})$ under $\mathrm{N}_{2}$ at $-10{ }^{\circ} \mathrm{C}$. The yellow solution was stirred at $-10{ }^{\circ} \mathrm{C}$ for $2 \mathrm{~h}$. The mixture was warmed to RT and stirred for an additional $1 \mathrm{~h}$. The solution was concentrated and the residue purified by flash column chromatography using a short column of silica, eluting with 1:1 hexanes-EtOAc, to give trans-5 as a colorless oil $(255 \mathrm{mg}, 91 \%) . \quad R_{f} 0.73(2: 1$ EtOAc-hexanes $) . \quad[\alpha]_{\mathrm{D}}{ }^{20}=-3.7^{\circ}(c 1.0$,

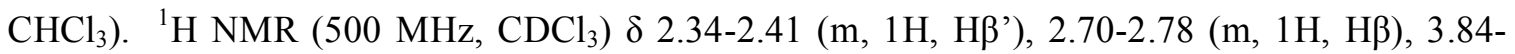
$3.93\left(\mathrm{~m}, 1.5 \mathrm{H}, \mathrm{H} \delta^{\prime}\right), 4.07$ (d, $\left.J=13.5 \mathrm{~Hz}, 0.5 \mathrm{H}, \mathrm{H} \delta\right), 4.17$ (t, $J=6.5 \mathrm{~Hz}, 0.5 \mathrm{H}$, Fmoc-CH), 4.28 (t, $J=6.5 \mathrm{~Hz}, 0.5 \mathrm{H}$, Fmoc-C므), 4.41-4.67 (m, 5H, Fmoc- $\left.\mathrm{CH}_{2}, \mathrm{C}_{2} \mathrm{CH}=\mathrm{CH}_{2}, \mathrm{H \alpha}\right), 5.21$ (d, $J=$ $\left.10.5 \mathrm{~Hz}, 0.5 \mathrm{H}, \mathrm{CH}=\mathrm{CH}_{2} c i s\right), 5.26\left(\mathrm{~d}, J=10.5 \mathrm{~Hz}, 0.5 \mathrm{H}, \mathrm{CH}=\mathrm{C}_{2} c i s\right), 5.27(\mathrm{~d}, J=17.8 \mathrm{~Hz}$, $0.5 \mathrm{H}, \mathrm{CH}=\mathrm{CH}_{2}$ trans $), 5.33\left(\mathrm{~d}, J=17.8 \mathrm{~Hz}, 0.5 \mathrm{H}, \mathrm{CH}=\underline{\mathrm{CH}}_{2}\right.$ trans $), 5.47(\mathrm{~d}, J=16.0 \mathrm{~Hz}, 1 \mathrm{H}, \mathrm{H} \gamma)$, 5.77-5.94 (m, 1H, $\left.\underline{\mathrm{C}}=\mathrm{CH}_{2}\right), 7.30$ (t, $\left.J=7.4 \mathrm{~Hz}, 2 \mathrm{H}, \mathrm{Ar}-\underline{\mathrm{H}}\right), 7.38$ (t, $\left.J=7.4 \mathrm{~Hz}, 2 \mathrm{H}, \mathrm{Ar}-\underline{\mathrm{H}}\right), 7.53-$ $7.61(\mathrm{~m}, 2 \mathrm{H}, \mathrm{Ar}-\underline{\mathrm{H}}), 7.75(\mathrm{~d}, J=7.2 \mathrm{~Hz}, 2 \mathrm{H}, \mathrm{Ar}-\underline{\mathrm{H}}) ;{ }^{13} \mathrm{C} \mathrm{NMR}\left(125 \mathrm{MHz}, \mathrm{CDCl}_{3}\right) \delta 36.4 \& 37.7$ $(\mathrm{C} \beta), 47.0 \& 47.2(\mathrm{Fmoc}-\underline{\mathrm{CH}}), 52.5 \& 52.9(\mathrm{C} \delta), 56.9 \& 57.3(\mathrm{C} \alpha), 66.3\left(\underline{\mathrm{CH}}_{2} \mathrm{CH}=\mathrm{CH}_{2}\right), 68.0(\mathrm{Fmoc}-$ $\left.\underline{\mathrm{CH}}_{2}\right), 85.5 \& 86.2(\mathrm{C} \gamma), 121.0\left(\mathrm{q}, J_{C-F}=320 \mathrm{~Hz}, \mathrm{SO}_{2} \underline{\mathrm{CF}}_{3}\right), 119.2 \& 119.5\left(\mathrm{CH}=\underline{\mathrm{CH}}_{2}\right), 120.0,124.9$, 127.1, 127.8, 131.1\&131 $\left(\underline{\mathrm{C}} \mathrm{H}=\mathrm{CH}_{2}\right), 141.3,143.6,154.0$ (Fmoc $\left.\underline{\mathrm{C}}=\mathrm{O}\right), 170.9$ (COOR). MS (MALDI-TOF, ACHA - $\alpha$-cyano-4-hydroxycinnamic acid) calcd for $\mathrm{MNa}^{+} \mathrm{C}_{24} \mathrm{H}_{22} \mathrm{~F}_{3} \mathrm{NNaO}_{7} \mathrm{~S}$ 548.10 , obsd 548.13. 


\section{$\mathrm{N}$-Fluorenylmethoxycarbonyl-cis-4-nitrobenzoyloxy-L-proline allyl ester (cis-9)}

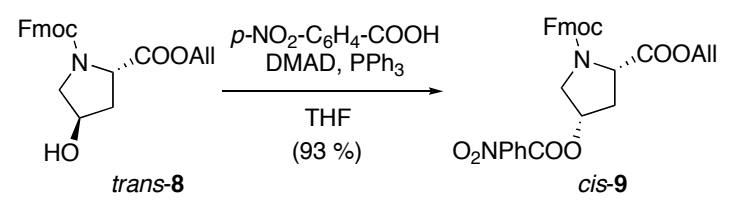

Dimethylazadicarboxylate (1.20 mL, $1.708 \mathrm{~g}, 11.70 \mathrm{mmol}, 2.20$ equiv.) was added dropwise (waiting for the fading of the yellow color after addition of each drop) to a solution of $\mathrm{N}$ fluorenylmethoxycarbonyl-trans-4-hydroxy-L-proline allyl ester (trans-8) (2.09 g, $5.32 \mathrm{mmol}$, 1.00 equiv.), triphenylphosphine (3.065 g, $11.70 \mathrm{mmol}, 2.20$ equiv.), and $p$-nitrobenzoic acid (1.776 g, $10.64 \mathrm{mmol}, 2.00$ equiv.) in dry THF $(100 \mathrm{~mL})$ under $\mathrm{N}_{2}$ at $0{ }^{\circ} \mathrm{C}$. The reaction was warmed to RT and stirred for $24 \mathrm{~h}$. The mixture was concentrated and the residue was partially purified by flash column chromatography, eluting with 3:1 hexanes-EtOAc. The residue was recrystallized from ether and a small amount of hexane to give cis-9 as a colorless crystalline solid (2.76 g, $93 \%) . \quad R_{f} 0.61$ (2:1 EtOAc-hexanes). $[\alpha]_{\mathrm{D}}{ }^{20}=-38.8^{\circ}\left(c 1.0, \mathrm{CHCl}_{3}\right) .{ }^{1} \mathrm{H} \mathrm{NMR}(500$

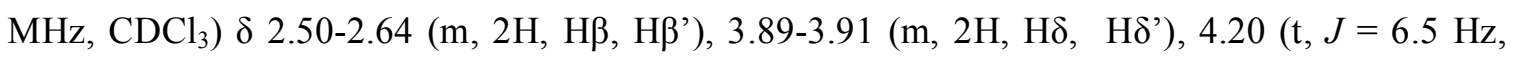
$0.5 \mathrm{H}$, Fmoc-CH), $4.28(\mathrm{t}, J=6.5 \mathrm{~Hz}, 0.5 \mathrm{H}$, Fmoc-C $\mathrm{H}), 4.37-4.70$ (m, 5H, Fmoc- $\mathrm{CH}_{2}$, $\left.\mathrm{C}_{2} \mathrm{CH}=\mathrm{CH}_{2}, \mathrm{H} \alpha\right), 5.08-5.29\left(\mathrm{~m}, 2 \mathrm{H}, \mathrm{CH}=\underline{\mathrm{CH}}_{2}\right), 5.62(\mathrm{~d}, J=27.2 \mathrm{~Hz}, 1 \mathrm{H}, \mathrm{H} \gamma), 5.71-5.85(\mathrm{~m}, 1 \mathrm{H}$, $\mathrm{C} \underline{\mathrm{H}}=\mathrm{CH}_{2}$ ), 7.29-7.35 (m, 2H, Ar- $\left.\underline{\mathrm{H}}\right)$, 7.39-7.46 (m, 2H, Ar- $\left.\underline{\mathrm{H}}\right)$, 7.57-7.65 (m, 2H, Ar- $\left.\underline{\mathrm{H}}\right), 7.78$ (d, $J=7.4 \mathrm{~Hz}, 2 \mathrm{H}, \mathrm{Ar}-\underline{\mathrm{H}}), 8.15\left(\mathrm{~d}, J=8.8 \mathrm{~Hz}, 1 \mathrm{H}, \mathrm{PNP}-\underline{\mathrm{H}}_{\text {arom }}\right), 8.19$ (d, $\left.J=8.8 \mathrm{~Hz}, 1 \mathrm{H}, \mathrm{PNP}-\underline{\mathrm{H}}_{\text {arom }}\right)$ $8.29\left(\mathrm{~d}, J=8.9 \mathrm{~Hz}, 1 \mathrm{H}, \mathrm{PNP}-\underline{H}_{\text {arom }}\right), 8.31$ (d, $\left.J=8.9 \mathrm{~Hz}, 1 \mathrm{H}, \mathrm{PNP}-\underline{H}_{\text {arom }}\right) ;{ }^{13} \mathrm{C} \mathrm{NMR}(125 \mathrm{MHz}$, $\left.\mathrm{CDCl}_{3}\right) \delta 35.6 \& 36.7(\mathrm{C} \beta), 47.1 \& 47.2($ Fmoc- $\mathrm{CH}), 52.4 \& 52.8(\mathrm{C} \delta), 57.5 \& 57.8(\mathrm{C} \alpha), 65.3$ $\left(\underline{\mathrm{CH}}_{2} \mathrm{CH}=\mathrm{CH}_{2}\right), 67.6 \& 67.7\left(\right.$ Fmoc- $\left.\underline{\mathrm{CH}}_{2}\right), 73.2 \& 74.3(\mathrm{C} \gamma), 118.6 \& 118.8\left(\mathrm{CH}=\underline{\mathrm{CH}}_{2}\right), 120.0,123.5$, 124.8, 127.0, 127.7, 130.9, 131.3\&131.5 $\left(\underline{\mathrm{CH}}=\mathrm{CH}_{2}\right), 141.2,143.7,150.7,154.3($ Fmoc $\underline{\mathrm{C}}=\mathrm{O})$, 163.8 (OㅁOPNB), 170.9 (ㅁOAll). HRMS (FAB+, NBA) calcd for $\mathrm{MH}^{+} \mathrm{C}_{30} \mathrm{H}_{26} \mathrm{~N}_{2} \mathrm{O}_{8} 543.1767$, obsd 543.1760 .

\section{$N$-Fluorenylmethoxycarbonyl-cis-4-hydroxy-L-proline allyl ester (cis-8)

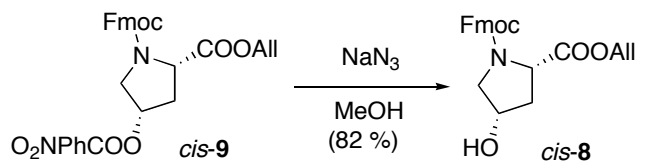

A solution of $\mathrm{N}$-fluorenylmethoxycarbonyl-cis-4-nitrobenzoyloxy-L-proline allyl ester (cis-9) (540 mg, $0.97 \mathrm{mmol}, 1.00$ equiv.) and sodium azide (234 mg, $3.59 \mathrm{mmol}, 3.71$ equiv.) in $\mathrm{MeOH}$ (36 mL) under $\mathrm{N}_{2}$ was stirred at $40{ }^{\circ} \mathrm{C}$ for $4 \mathrm{~h}$. The solvent was removed and the residue purified by flash column chromatography, eluting with 3:1 hexanes-EtOAc to 1:1 hexanes-EtOAc, to give cis-8 (310 mg, $82 \%) . \quad R_{f} 0.61\left(2: 1\right.$ EtOAc-hexanes). $[\alpha]_{\mathrm{D}}{ }^{20}=-56.1^{\circ}\left(c 1.0, \mathrm{CHCl}_{3}\right) .{ }^{1} \mathrm{H} \mathrm{NMR}$ 
$\left(500 \mathrm{MHz}, \mathrm{CDCl}_{3}\right) \delta 2.14-2.20(\mathrm{~m}, 1 \mathrm{H}, \mathrm{H} \beta$ '), 2.32-2.40 (m, 1H, H $\beta), 3.00(\mathrm{~d}, J=8.8 \mathrm{~Hz}, 0.5 \mathrm{H}$,

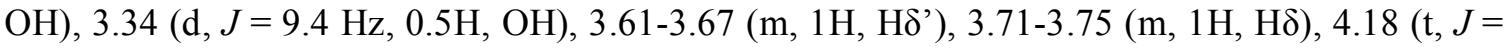
$6.6 \mathrm{~Hz}, 0.5 \mathrm{H}, \mathrm{Fmoc}-\mathrm{CH}), 4.25$ (t, $J=7.1 \mathrm{~Hz}, 0.5 \mathrm{H}, \mathrm{Fmoc}-\mathrm{C} \underline{\mathrm{H}}), 4.33-4.61\left(\mathrm{~m}, 6 \mathrm{H}, \mathrm{CH}_{2} \mathrm{CH}=\mathrm{CH}_{2}\right.$, Fmoc- $\left.\mathrm{CH}_{2}, \mathrm{H} \gamma, \mathrm{H} \alpha\right), 5.19-5.38\left(\mathrm{~m}, 2 \mathrm{H}, \mathrm{CH}=\mathrm{CH}_{2}\right), 5.79-5.96\left(\mathrm{~m}, 1 \mathrm{H}, \mathrm{C} \underline{H}=\mathrm{CH}_{2}\right), 7.29-7.32(\mathrm{~m}$, $2 \mathrm{H}, \operatorname{Ar}-\underline{\mathrm{H}}), 7.38-7.41$ (m, 2H, Ar- $\underline{\mathrm{H}}), 7.54-7.61$ (m, 2H, Ar- $\underline{\mathrm{H}}), 7.75-7.77$ (m, 2H, Ar- $\underline{\mathrm{H}}) ;{ }^{13} \mathrm{C}$ NMR $\left(125 \mathrm{MHz}, \mathrm{CDCl}_{3}\right) \delta 37.7 \& 38.8(\mathrm{C} \beta), 47.0 \& 47.1$ (Fmoc- $\left.\mathrm{CH}\right), 55.5 \& 56.0(\mathrm{C} \delta), 57.6 \& 58.1$ $(\mathrm{C} \alpha), 66.3 \& 66.4\left(\underline{\mathrm{CH}}_{2} \mathrm{CH}=\mathrm{CH}_{2}\right), 67.6\left(\right.$ Fmoc- $\left.\underline{\mathrm{CH}}_{2}\right), 69.9 \& 71.1(\mathrm{C} \gamma), 118.9 \& 119.2\left(\mathrm{CH}=\underline{\mathrm{CH}}_{2}\right)$, $119.9,125.0,127.1,127.7,131.1 \& 131.2\left(\underline{\mathrm{CH}}=\mathrm{CH}_{2}\right), 141.3,143.6,154.7($ Fmoc $\underline{\mathrm{C}}=\mathrm{O}), 173.9$ (ㅁOR). HRMS (FAB+, NBA) calcd for $\mathrm{MH}^{+} \mathrm{C}_{23} \mathrm{H}_{24} \mathrm{NO}_{5} 394.1655$, obsd 394.1654.

\section{$\mathrm{N}$-Fluorenylmethoxycarbonyl-cis-4-trifluoromethylsulfonyloxy-L-proline allyl ester (cis-5)}

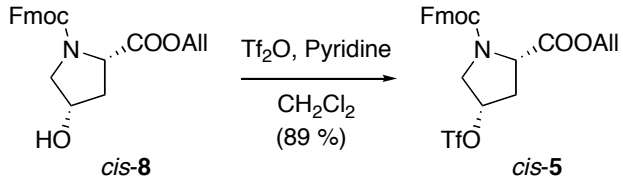

By analogy to the preparation of trans $-\mathbf{5}$ on a scale of $0.78 \mathrm{mmol}$ to give cis $\mathbf{- 5}$ as a colorless oil (364 mg, $89 \%)$. $R_{f} 0.68$ (2:1 EtOAc-hexanes). $[\alpha]_{\mathrm{D}}{ }^{20}=-24.3^{\circ}\left(c 1.0, \mathrm{CHCl}_{3}\right) .{ }^{1} \mathrm{H}$ NMR (500

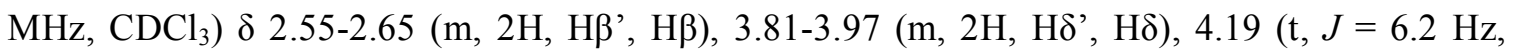
0.5H, Fmoc-CH), $4.29(\mathrm{t}, J=6.7 \mathrm{~Hz}, 0.5 \mathrm{H}$, Fmoc-C $\mathrm{H}), 4.35-4.73$ (m, 5H, Fmoc- $\mathrm{CH}_{2}$, $\left.\mathrm{C}_{2} \mathrm{CH}=\mathrm{CH}_{2}, \mathrm{H} \alpha\right), 5.21-5.34\left(\mathrm{~m}, 2 \mathrm{H}, \mathrm{CH}=\mathrm{C}_{2}\right), 5.46(\mathrm{~d}, J=10.8 \mathrm{~Hz}, 1 \mathrm{H}, \mathrm{H} \gamma), 5.80-5.93(\mathrm{~m}, 1 \mathrm{H}$, $\left.\mathrm{C} \underline{\mathrm{H}}=\mathrm{CH}_{2}\right), 7.28-7.33$ (m, 2H, Ar- $\left.\underline{\mathrm{H}}\right), 7.37-7.40$ (m, 2H, Ar- $\left.\underline{\mathrm{H}}\right), 7.51-7.59$ (m, 2H, Ar- $\left.\underline{\mathrm{H}}\right), 7.76$ (t, $J$ $=7.7 \mathrm{~Hz}, 2 \mathrm{H}, \mathrm{Ar}-\underline{\mathrm{H}}) .{ }^{13} \mathrm{C}$ NMR $\left(125 \mathrm{MHz}, \mathrm{CDCl}_{3}\right) \delta 36.5 \& 37.8(\mathrm{C} \beta), 47.1 \& 47.2$ (Fmoc- $\left.\underline{\mathrm{H}}\right)$ ), $52.4 \& 52.9(\mathrm{C} \delta), 57.2 \& 57.5(\mathrm{C \alpha}), 66.4 \& 66.5\left(\mathrm{CH}_{2} \mathrm{CH}=\mathrm{CH}_{2}\right), 67.8 \& 67.9\left(\mathrm{Fmoc}-\mathrm{CH}_{2}\right), 85.5 \& 86.3$ $(\mathrm{C} \gamma), 117.1\left(\mathrm{q}, J_{C-F}=320 \mathrm{~Hz}, \mathrm{SO}_{2} \underline{\mathrm{CF}}_{3}\right), 119.2 \& 119.5\left(\mathrm{CH}=\underline{\mathrm{CH}_{2}}\right), 131.1 \& 131\left(\underline{\mathrm{CH}}=\mathrm{CH}_{2}\right),[120.0$, 124.9, 127.1, 127.8, 141.3, 143.6] (Ar-4oㄷ), 154.0 (Fmoc $\underline{\mathrm{C}}=\mathrm{O}), 169.8$ (ㅁOR). MS (MALDITOF, ACHA) for $\mathrm{C}_{24} \mathrm{H}_{22} \mathrm{~F}_{3} \mathrm{NO}_{7} \mathrm{~S}\left(\mathrm{MNa}^{+}\right)$548.10, obsd 548.11.

\section{4-O-(3,4,6-Tri- $O$-benzyl- $\beta$-D-mannopyranosyl)- $N$-fluorenylmethoxycarbonyl-trans-4- hydroxy-L-proline allyl ester (trans-4)}

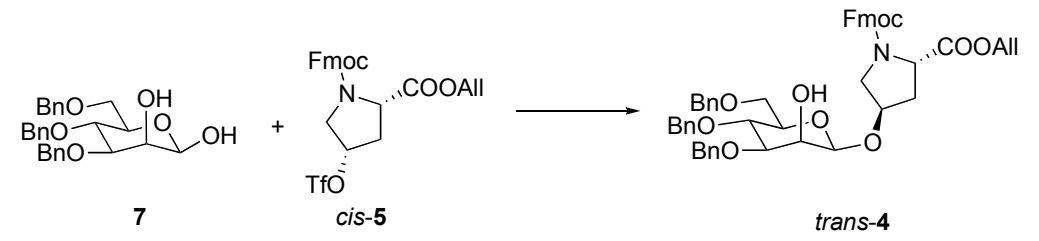

Dibutyltin oxide (122 mg, $0.49 \mathrm{mmol}, 2.1$ equiv.) was added to a solution of 3,4,6-tri- $O$-benzylD-mannopyranose (7) (210 $\mathrm{mg}, 0.47 \mathrm{mmol}, 2.0$ equiv.) in dry toluene (5 mL) under $\mathrm{N}_{2}$. The 
mixture was stirred at $60{ }^{\circ} \mathrm{C}$ for $3 \mathrm{~h}$. Cesium fluoride (77 mg, $0.47 \mathrm{mmol}, 2.0$ equiv.) was added and the mixture was concentrated under vacuum for $2 \mathrm{~h}$. With minimal exposure to air, flamedried molecular sieves were added and the residue was suspended in $\mathrm{CH}_{2} \mathrm{Cl}_{2}(3 \mathrm{~mL})$ under $\mathrm{N}_{2}$ then cooled in an ice-salt bath. $N$-Fluorenylmethoxycarbonyl-trans-4-trifluoromethylsulfonyloxyL-proline allyl ester (cis-5) (122 mg, $0.23 \mathrm{mmol}, 1.0$ equiv.) in $\mathrm{CH}_{2} \mathrm{Cl}_{2}(2 \mathrm{~mL})$ was added and the solution was stirred at RT overnight $(18 \mathrm{~h})$. The reaction mixture was diluted with $\mathrm{CH}_{2} \mathrm{Cl}_{2}(100$ $\mathrm{mL})$, washed with water $(100 \mathrm{~mL})$, dried over $\mathrm{MgSO}_{4}$, filtered, and concentrated under vacuum. The residue was purified by flash column chromatography, eluting with 2:1 hexanes-EtOAc, to give trans-4 as a colorless solid (115 mg, $60 \%) . R_{f} 0.58\left(2: 1\right.$ EtOAc-hexanes). $[\alpha]_{\mathrm{D}}^{20}=-30.2^{\circ}(c$

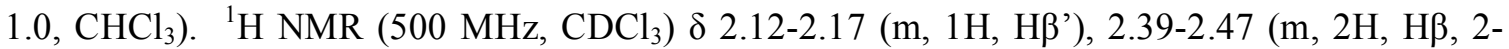

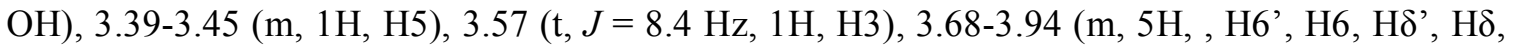
H4), 4.07 (s, 1H, H2), 4.15 (t, $J=6.8 \mathrm{~Hz}, 0.5 \mathrm{H}, \mathrm{Fmoc}-\mathrm{CH}), 4.22$ (t, $J=7.8 \mathrm{~Hz}, 0.5 \mathrm{H}$, Fmoc-C $\underline{\mathrm{H}}$ ), 4.29-4.69 (m, 11H, H1, H $\gamma, \mathrm{H} \alpha, 2$ x $\mathrm{PhCH}_{2} \mathrm{O}, \mathrm{CH}_{2} \mathrm{CH}=\mathrm{CH}_{2}, \mathrm{Fmoc}_{-} \mathrm{CH}_{2}$ ), 4.75-4.90 (m, 2H, $\left.\mathrm{PhC}_{2} \mathrm{O}\right), 5.17-5.34\left(\mathrm{~m}, 2 \mathrm{H}, \mathrm{CH}=\mathrm{C}_{2}\right), 5.78-5.94\left(\mathrm{~m}, 1 \mathrm{H}, \mathrm{C} \underline{\mathrm{H}}=\mathrm{CH}_{2}\right), 7.20-7.40(\mathrm{~m}, 19 \mathrm{H}, \mathrm{Ar}-\underline{\mathrm{H}}$ $\mathrm{x} 4, \mathrm{Ph}-\underline{\mathrm{H}}), 7.53-7.60(\mathrm{~m}, 2 \mathrm{H}, \mathrm{Ar}-\underline{\mathrm{H}}), 7.75(\mathrm{~d}, J=7.5 \mathrm{~Hz}, 2 \mathrm{H}, \mathrm{Ar}-\underline{\mathrm{H}}) ;{ }^{13} \mathrm{C}$ NMR $(125 \mathrm{MHz}$, $\left.\mathrm{CDCl}_{3}\right) \delta 34.9 \& 36.2(\mathrm{C} \beta), 47.0 \& 47.2(\mathrm{Fmoc}-\underline{\mathrm{CH}}), 52.8 \& 53.2(\mathrm{C} \delta), 57.4 \& 57.7(\mathrm{C} \alpha), 65.8 \& 65.9$ $\left(\underline{\mathrm{CH}}_{2} \mathrm{CH}=\mathrm{CH}_{2}\right), 67.6 \& 67.7 \quad\left(\mathrm{Fmoc}-\underline{\mathrm{CH}} \mathrm{H}_{2}\right), 68.3(\mathrm{C} 2), 69.0 \& 69.1(\mathrm{C} 6), 71.5\left(\mathrm{PhCH}_{2} \mathrm{O}\right), 73.5$ $\left(\mathrm{PhCH}_{2} \mathrm{O}\right), 74.0$ (C4), 75.1 (C5), $75.4\left(\mathrm{PhCH}_{2} \mathrm{O}\right), 76.0(\mathrm{C} \gamma), 81.3$ (C3), 98.3\&98.6 (C1), $118.6 \& 118.9\left(\mathrm{CH}=\underline{\mathrm{CH}}_{2}\right), 119.9,124.9,125.1,125.2,127.1,127.6,127.7,127.8,127.9,128.1$, $128.3,128.4,128.5,131.4 \& 131.6\left(\underline{\mathrm{CH}}=\mathrm{CH}_{2}\right), 137.6,137.7,138.0,138.1,141.1,143.6,143.8$, 143.9, 144.1, 154.8 (Fmoc $\underline{\mathrm{C}}=\mathrm{O}), 171.9 \& 172.0$ (COOR). HRMS (FAB+, NBA) calcd for $\mathrm{MH}^{+}$ $\mathrm{C}_{50} \mathrm{H}_{52} \mathrm{NO}_{10} 826.3591$, obsd 826.3580,

\section{4-O-(3,4,6-Tri- $O$-benzyl- $\beta$-D-mannopyranosyl)- $N$-fluorenylmethoxycarbonyl-cis-4-hydroxy-}

\section{L-proline allyl ester (cis-4)}

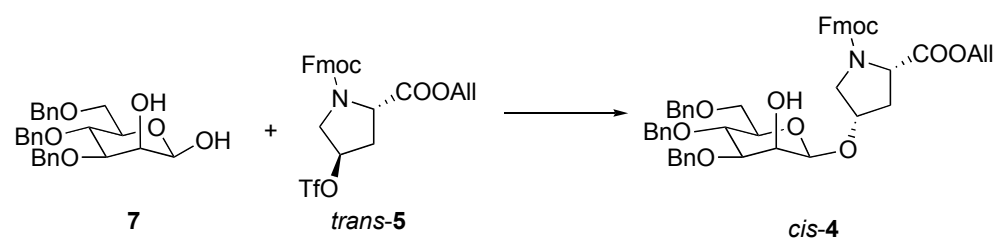

By analogy to the procedure for the preparation of trans -4 on a scale of $0.404 \mathrm{mmol}$ to give cis- $\mathbf{4}$ as a colorless solid (96 mg, $57 \%$ ). $R_{f} 0.48$ (2:1 EtOAc-hexanes). $[\alpha]_{\mathrm{D}}{ }^{20}=-29.6^{\circ}\left(c 1.0, \mathrm{CHCl}_{3}\right)$.

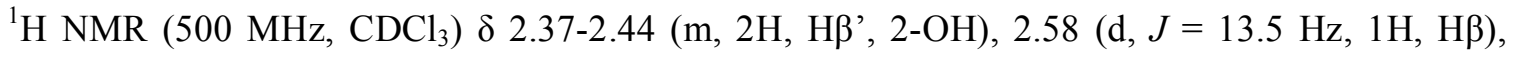

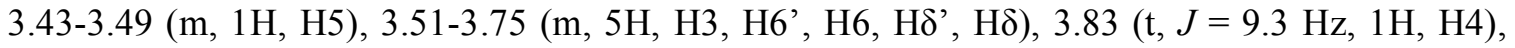
$4.00(\mathrm{dd}, J=18.5,2.8 \mathrm{~Hz}, 1 \mathrm{H}, \mathrm{H} 2), 4.18$ (t, $J=6.6 \mathrm{~Hz}, 0.5 \mathrm{H}, \mathrm{Fmoc}-\mathrm{CH}), 4.27$ (t, $J=7.1 \mathrm{~Hz}$, 


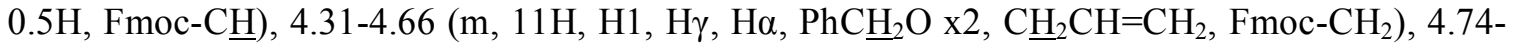
$4.91\left(\mathrm{~m}, 2 \mathrm{H}, \mathrm{PhC}_{2} \mathrm{O}\right), 5.16-5.31\left(\mathrm{~m}, 2 \mathrm{H}, \mathrm{CH}=\underline{\mathrm{C}}_{2}\right), 5.81-5.93\left(\mathrm{~m}, 1 \mathrm{H}, \mathrm{C} \underline{\mathrm{H}}=\mathrm{CH}_{2}\right), 7.18-7.39(\mathrm{~m}$, 19H, Ar- $\underline{\mathrm{H}} \mathrm{x} 4, \mathrm{Ph}-\underline{\mathrm{H}}), 7.57-7.67$ (m, 2H, Ar- $\underline{\mathrm{H}}), 7.79$ (m, $J=7.0 \mathrm{~Hz}, 2 \mathrm{H}, \mathrm{Ar}-\underline{\mathrm{H}}) ;{ }^{13} \mathrm{C} \mathrm{NMR}(125$ $\left.\mathrm{MHz}, \mathrm{CDCl}_{3}\right) \delta 36.9 \& 37.8(\mathrm{C} \beta), 47.0 \& 47.1$ (Fmoc- $\left.\mathrm{CH}\right), 51.6 \& 51.9(\mathrm{C} \delta), 57.6 \& 57.9(\mathrm{C} \alpha)$, $65.8 \& 65.9\left(\underline{\mathrm{CH}}_{2} \mathrm{CH}=\mathrm{CH}_{2}\right), 67.4\left(\mathrm{Fmoc}-\underline{\mathrm{CH}}_{2}\right), 68.0(\mathrm{C} 2), 69.2 \& 69.3(\mathrm{C} 6), 71.1\left(\mathrm{PhCH}_{2} \mathrm{O}\right), 73.3$ ( $\left.\mathrm{Ph}^{-} \mathrm{H}_{2} \mathrm{O}\right), 73.8(\mathrm{C} 4), 74.9$ (C5), $75.0\left(\mathrm{PhCH}_{2} \mathrm{O}\right), 76.4(\mathrm{C} \gamma), 80.9 \& 81.0(\mathrm{C} 3), 97.9 \& 98.2(\mathrm{C} 1)$, $118.9 \& 119.2\left(\mathrm{CH}=\underline{\mathrm{C}} \mathrm{H}_{2}\right), 120.0,124.9,125.0,125.2,127.1,127.7,127.8,127.9,128.1,128.2$, $128.4,128.5,131.1 \& 131.2\left(\underline{\mathrm{C}} \mathrm{H}=\mathrm{CH}_{2}\right), 137.8,137.9,138.2,141.3,143.4,143.7,143.8,144.2$, $154.6 \& 154.7$ (Fmoc $\underline{\mathrm{C}}=\mathrm{O}), 171.5 \& 171.6$ ( $\underline{\mathrm{COOR}})$. HRMS (FAB+, NBA) calcd for $\mathrm{MH}^{+}$ $\mathrm{C}_{50} \mathrm{H}_{52} \mathrm{NO}_{10} 826.3591$, obsd 826.3593.

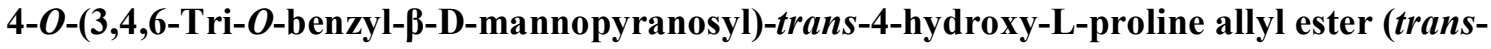

14)

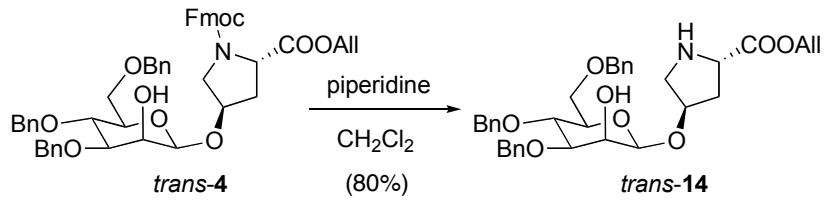

4-O-(3,4,6-Tri-O-benzyl- $\beta$-D-mannopyranosyl)- $N$-fluorenylmethoxycarbonyl-cis-4-hydroxy- $L$ proline allyl ester (trans-4) $(69 \mathrm{mg}, 0.084 \mathrm{mmol})$ was dissolved in a mixture of piperidine (100 $\mu \mathrm{L})$ and $\mathrm{CH}_{2} \mathrm{Cl}_{2}(2 \mathrm{~mL})$ and stirred under $\mathrm{N}_{2}$ for $1 \mathrm{~h}$. The mixture was diluted with $\mathrm{CH}_{2} \mathrm{Cl}_{2}(50$ $\mathrm{mL})$, washed with water $(50 \mathrm{~mL})$, dried over $\mathrm{MgSO}_{4}$, filtered, and concentrated. The residue was purified by flash column chromatography, eluting with $1-4 \% \mathrm{MeOH}$ in $\mathrm{CH}_{2} \mathrm{Cl}_{2}$ to give trans-14 (40 mg, $80 \%$ \%). $R_{f} 0.26\left(9: 1 \mathrm{CH}_{2} \mathrm{Cl}_{2}-\mathrm{MeOH}\right) .[\alpha]_{\mathrm{D}}{ }^{20}=-30.5^{\circ}\left(c 1.0, \mathrm{CHCl}_{3}\right) .{ }^{1} \mathrm{H} \mathrm{NMR}(500 \mathrm{MHz}$,

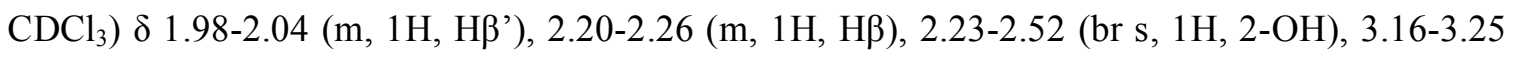

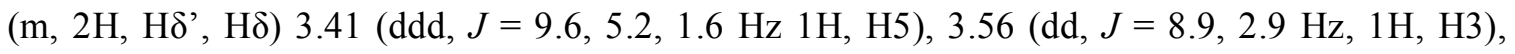

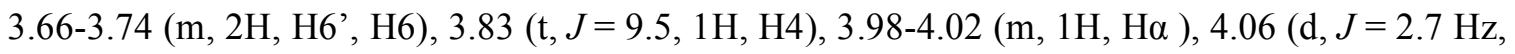
$1 \mathrm{H}, \mathrm{H} 2), 4.46-4.68$ (m, 8H, H1, $\left.\mathrm{H} \gamma, 2 \times \mathrm{PhC}_{2} \mathrm{O}, \underline{\mathrm{C}}_{2} \mathrm{CH}=\mathrm{CH}_{2}\right), 4.75-4.89$ (m, 2H, $\left.\mathrm{PhC}_{2} \mathrm{O}\right)$, 5.24-5.34 (m, 2H, $\left.\mathrm{CH}=\mathrm{CH}_{2}\right), 5.87-5.95\left(\mathrm{~m}, 1 \mathrm{H}, \mathrm{CH}=\mathrm{CH}_{2}\right), 7.18-7.39(\mathrm{~m}, 15 \mathrm{H}, \mathrm{Ph}-\underline{\mathrm{H}}) ;{ }^{13} \mathrm{C} \mathrm{NMR}$ $\left(125 \mathrm{MHz}, \mathrm{CDCl}_{3}\right) \delta 36.4(\mathrm{C} \beta), 53.6(\mathrm{C} \delta), 58.5(\mathrm{C} \alpha), 65.6\left(\mathrm{CH}_{2} \mathrm{CH}=\mathrm{CH}_{2}\right), 68.5(\mathrm{C} 2), 69.2(\mathrm{C} 6)$,

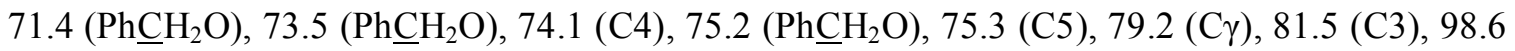
(C1), $118.7\left(\mathrm{CH}=\underline{\mathrm{CH}}_{2}\right), 127.6,127.7,127.8,127.9,128.1,128.4,128.5,131.8\left(\underline{\mathrm{CH}}=\mathrm{CH}_{2}\right), 174.9$ (COOR). HRMS (FAB+, NBA) calcd for $\mathrm{MH}^{+} \mathrm{C}_{35} \mathrm{H}_{42} \mathrm{NO}_{8}$ 604.2910, obsd 604.2906. 


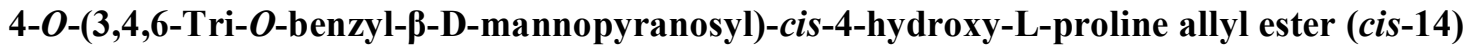

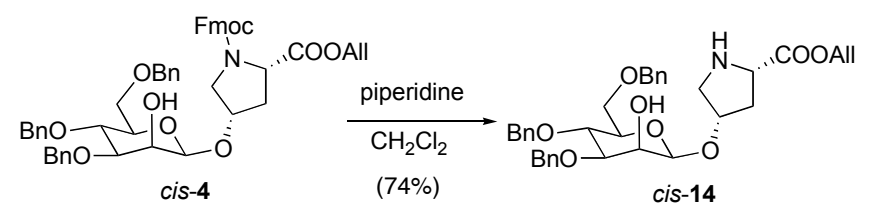

By analogy to the preparation for trans $\mathbf{- 1 4}$ on a scale of $0.084 \mathrm{mmol}$ to give cis-14 (37 $\mathrm{mg}, 74 \%$ ). $R_{f} 0.50\left(9: 1 \mathrm{CH}_{2} \mathrm{Cl}_{2}-\mathrm{MeOH}\right) .[\alpha]_{\mathrm{D}}{ }^{20}=-30.3^{\circ}\left(c\right.$ 1.0, $\left.\mathrm{CHCl}_{3}\right) .{ }^{1} \mathrm{H} \mathrm{NMR}\left(500 \mathrm{MHz}, \mathrm{CDCl}_{3}\right) \delta 2.34-$

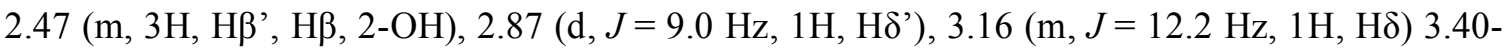
3.43 (m, 1H, H5), 3.48 (dd, $J=9.0,3.1 \mathrm{~Hz}, 1 \mathrm{H}, \mathrm{H} 3), 3.65-3.85$ (m, 3H, H6', H6, H4), 4.00 (d, $J=$ $2.7 \mathrm{~Hz}, 1 \mathrm{H}, \mathrm{H} 2), 4.41-4.66$ (m, 9H, H1, $\mathrm{H} \gamma, \mathrm{H} \alpha, 2$ x $\left.\mathrm{PhCH}_{2} \mathrm{O}, \mathrm{C}_{2} \mathrm{CH}=\mathrm{CH}_{2}\right), 4.74-4.91$ (m, 2H, $\left.\mathrm{PhC}_{\mathrm{H}_{2}} \mathrm{O}\right), 5.16-5.31\left(\mathrm{~m}, 2 \mathrm{H}, \mathrm{CH}=\underline{\mathrm{C}}_{2}\right), 5.81-5.93\left(\mathrm{~m}, 1 \mathrm{H}, \mathrm{C} \underline{\mathrm{H}}=\mathrm{CH}_{2}\right), 7.18-7.39(\mathrm{~m}, 15 \mathrm{H}, \mathrm{Ph}-\underline{\mathrm{H}})$; ${ }^{13} \mathrm{C}$ NMR (125 MHz, $\left.\mathrm{CDCl}_{3}\right) \delta 37.9(\mathrm{C} \beta), 52.1(\mathrm{C} \delta), 65.2(\mathrm{C} \alpha), 65.9\left(\mathrm{CH}_{2} \mathrm{CH}=\mathrm{CH}_{2}\right), 68.3(\mathrm{C} 2)$, 69.3 (C6), $71.2\left(\mathrm{PhCH}_{2} \mathrm{O}\right), 73.4\left(\mathrm{PhCH}_{2} \mathrm{O}\right), 74.0$ (C4), 75.0 (C5), $75.1\left(\mathrm{PhCH}_{2} \mathrm{O}\right), 76.2$ (C $\left.\gamma\right), 81.2$ (C3), $97.9(\mathrm{C} 1), 118.6\left(\mathrm{CH}=\underline{\mathrm{CH}}_{2}\right), 127.6,127.6,128.8,128.1,128.3,128.4,131.9\left(\underline{\mathrm{CH}}=\mathrm{CH}_{2}\right)$, 137.8, 138.2, 173.3 (COOR). HRMS (FAB+, NBA) calcd for $\mathrm{MH}^{+} \mathrm{C}_{35} \mathrm{H}_{42} \mathrm{NO}_{8}$ 604.2910, obsd 604.2908 .

\section{4- $O$-(3,4,6-Tri- $O$-benzyl-2- $O$-trifluromethanesulfonyl- $\beta$-D-mannopyranosyl)- $N$ -}

fluorenylmethoxycarbonyl-trans-4-hydroxy-L-proline allyl ester (15)

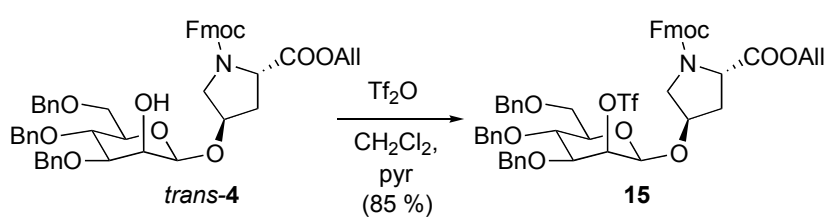

Triflic anhydride (31 $\mu \mathrm{L}, 51 \mathrm{mg}, 0.182 \mathrm{mmol}, 1.5$ equiv.) was added to a solution of 4-O-(3,4,6tri-O-benzyl- $\beta$-D-mannopyranosyl)- $N$-fluorenylmethoxycarbonyl-trans-4-hydroxy-L-proline allyl ester (trans-4) (100 mg, $0.121 \mathrm{mmol}, 1.0$ equiv.) in a mixture of $\mathrm{CH}_{2} \mathrm{Cl}_{2}(2 \mathrm{~mL})$ and pyridine (1 $\mathrm{mL}$ ) at $-10^{\circ} \mathrm{C}$ under $\mathrm{N}_{2}$. The mixture was stirred for 30 min at $0{ }^{\circ} \mathrm{C}$ then allowed to warm to RT and then stirred for $1.5 \mathrm{~h}$. The mixture was diluted with $\mathrm{CH}_{2} \mathrm{Cl}_{2}(100 \mathrm{~mL})$, washed with water $(100 \mathrm{~mL})$, dried over $\mathrm{MgSO}_{4}$, filtered, and concentrated. The residue was purified by flash column chromatography, eluting with 2:1 hexanes-EtOAc, to give $\mathbf{1 5}$ as a yellow oil (98 $\mathrm{mg}, 85$ \%). $R_{f} 0.67$ (2:1 EtOAc-hexanes). $[\alpha]_{\mathrm{D}}{ }^{20}=-37.0^{\circ}$ (c 1.0, $\left.\mathrm{CHCl}_{3}\right) .{ }^{1} \mathrm{H}$ NMR (500 MHz, $\left.\mathrm{CDCl}_{3}\right) \delta$

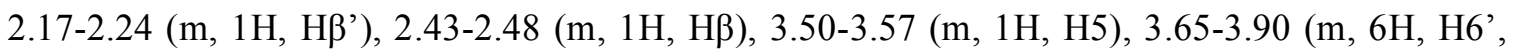

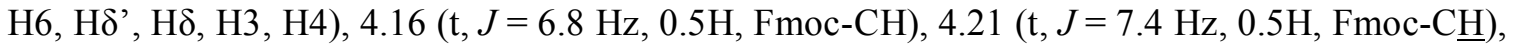
4.28-4.67 (m, $10 \mathrm{H}, \mathrm{H} 1, \mathrm{H} \gamma, \mathrm{H \alpha}, 2$ x $\left.\mathrm{PhC}_{2} \mathrm{O}, \underline{\mathrm{C}}_{2} \mathrm{CH}=\mathrm{CH}_{2}, \mathrm{Fmoc}_{-} \mathrm{CH}_{2}\right), 4.80-4.87$ (m, 2H, $\left.\mathrm{PhC}_{2} \mathrm{O}\right), 5.12(\mathrm{dd}, J=13.4,2.4 \mathrm{~Hz} 1 \mathrm{H}, \mathrm{H} 2), 5.18-5.35\left(\mathrm{~m}, 2 \mathrm{H}, \mathrm{CH}=\underline{\mathrm{C}}_{2}\right), 5.79-5.95(\mathrm{~m}, 1 \mathrm{H}$, 
$\left.\mathrm{C} \underline{\mathrm{H}}=\mathrm{CH}_{2}\right), 7.15-7.40(\mathrm{~m}, 19 \mathrm{H}, \mathrm{Ar}-\underline{\mathrm{H}}), 7.59-7.66(\mathrm{~m}, 2 \mathrm{H}, \mathrm{Ar}-\underline{\mathrm{H}}), 7.79(\mathrm{~d}, J=7.4 \mathrm{~Hz}, 2 \mathrm{H}, \mathrm{Ar}-\underline{\mathrm{H}})$. ${ }^{13} \mathrm{C}$ NMR $\left(125 \mathrm{MHz}, \mathrm{CDCl}_{3}\right) \delta 35.2 \& 36.7(\mathrm{C} \beta), 47.0 \& 47.2$ (Fmoc- $\left.\underline{\mathrm{CH}}\right), 52.9 \& 53.3(\mathrm{C} \delta)$, $57.3 \& 57.9(\mathrm{C} \alpha), 65.8 \& 65.9\left(\underline{\mathrm{CH}}_{2} \mathrm{CH}=\mathrm{CH}_{2}\right), 67.7 \& 67.8\left(\right.$ Fmoc- $\left.\underline{\mathrm{CH}_{2}}\right), 68.8(\mathrm{C} 6), 72.5\left(\mathrm{PhCH}_{2} \mathrm{O}\right)$,

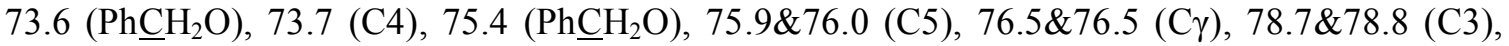
82.1\&82.2 (C2), 96.0\&96.3 (C1), 118.7\&119.0 (CH= $\left.\underline{\mathrm{CH}}_{2}\right), 119.9\left(\mathrm{q}, J_{C-F}=320 \mathrm{~Hz}, \mathrm{SO}_{2} \underline{\mathrm{CF}}_{3}\right)$, $119.8,119.9,125.0,125.2$, 125.3, 127.1, 127.7, 127.9, 128.0, 128.1, 128.2, 128.4, 128.5, 128.6, $129.1,131.5 \& 131.7\left(\underline{\mathrm{C}} \mathrm{H}=\mathrm{CH}_{2}\right), 136.8,136.9,137.6,137.7,137.9,138.1,141.3,143.9,144.0$, 144.2, 154.2\&154.8 (Fmoc $\underline{\mathrm{C}}=\mathrm{O}), 172.0$ ( $\underline{\mathrm{COOR}}$ ). HRMS (FAB+, NBA) calcd for $\mathrm{MH}^{+}$ $\mathrm{C}_{51} \mathrm{H}_{51} \mathrm{~F}_{3} \mathrm{NO}_{12} \mathrm{~S}$ 958.3084, obsd 958.3067.

\section{4-O-(2-Azido-3,4,6-tri-O-benzyl-2-deoxy- $\beta$-D-glucopyranosyl)- $\mathrm{N}$-fluorenylmethoxycarbonyl- trans-4-hydroxy-L-proline allyl ester (16)

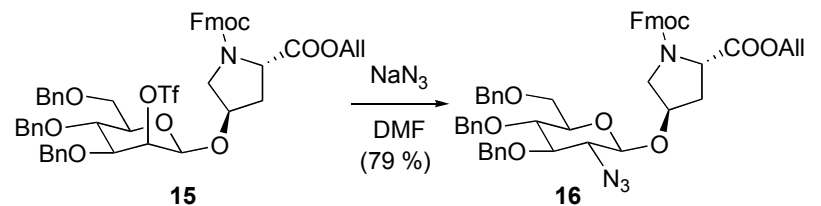

Sodium azide ( $8 \mathrm{mg}, 0.111 \mathrm{mmol}, 2.0$ equiv.) was added to a solution of 4-O-(3,4,6-tri- $O$-benzyl2-O-trifluromethanesulfonyl- $\beta$-D-mannopyranosyl)- $N$-fluorenylmethoxycarbonyl-trans-4hydroxy-L-proline allyl ester (15) $(53 \mathrm{mg}, 0.055 \mathrm{mmol}, 1.0$ equiv.) in dry DMF (2 mL) and the solution was stirred at RT under $\mathrm{N}_{2}$ for $2 \mathrm{~h}$. The mixture was diluted with $\mathrm{CH}_{2} \mathrm{Cl}_{2}(100 \mathrm{~mL})$, washed with water $(2 \times 100 \mathrm{~mL})$, filtered, dried over $\mathrm{MgSO}_{4}$, and concentrated. The residue was purified by flash column chromatography, eluting with 2:1 hexanes-EtOAc, to give $\mathbf{1 6}$ as a colorless oil (37 mg, $79 \%) . \quad R_{f} 0.75$ (2:1 EtOAc-hexanes). $[\alpha]_{\mathrm{D}}{ }^{20}=-34.5^{\circ}\left(c 1.0, \mathrm{CHCl}_{3}\right) .{ }^{1} \mathrm{H}$

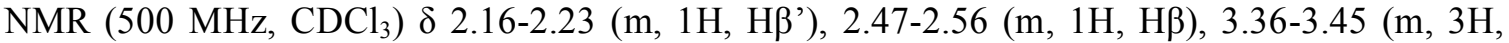

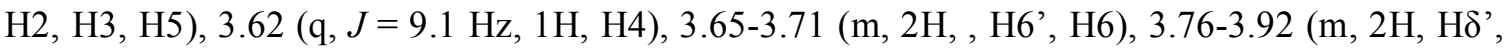
$\mathrm{H} \delta$ ), 4.16 (t, $J=6.9 \mathrm{~Hz}, 0.5 \mathrm{H}$, Fmoc-CH), 4.24 (t, $J=7.5 \mathrm{~Hz}, 0.5 \mathrm{H}$, Fmoc-C마), 4.30-4.67 (m, $10 \mathrm{H}, \mathrm{H} 1, \mathrm{H} \gamma, \mathrm{H} \alpha, 1.5$ x $\left.\mathrm{PhC}_{\mathrm{H}_{2}} \mathrm{O}, \mathrm{C}_{2} \mathrm{CH}=\mathrm{CH}_{2}, \mathrm{Fmoc}-\mathrm{CH}_{2}\right), 4.77-4.88$ (m, 3H, $1.5 \times \mathrm{PhCH}_{2} \mathrm{O}$ ), 5.19-5.36 (m, 2H, $\left.\mathrm{CH}=\mathrm{CH}_{2}\right), 5.80-5.96\left(\mathrm{~m}, 1 \mathrm{H}, \mathrm{CH}=\mathrm{CH}_{2}\right), 7.15-7.46(\mathrm{~m}, 19 \mathrm{H}, \mathrm{Ar}-\underline{\mathrm{H}}), 7.59$ (dd, $J$ $=22.9,7.4 \mathrm{~Hz}, 2 \mathrm{H}, \mathrm{Ar}-\underline{\mathrm{H}}), 7.75(\mathrm{dd}, J=7.4,3.0 \mathrm{~Hz}, 2 \mathrm{H}, \mathrm{Ar}-\underline{\mathrm{H}}) ;{ }^{13} \mathrm{C}$ NMR $\left(125 \mathrm{MHz}, \mathrm{CDCl}_{3}\right) \delta$ $35.4 \& 36.7(\mathrm{C} \beta), 47.1 \& 47.2($ Fmoc- $\underline{\mathrm{CH}}), 52.8 \& 53.3(\mathrm{C} \delta), 57.5 \& 57.8(\mathrm{C} \alpha), 65.9\left(\underline{\mathrm{C}} \mathrm{H}_{2} \mathrm{CH}=\mathrm{CH}_{2}\right)$, 66.0 (C2), $67.7\left(\right.$ Fmoc- $\left.\underline{-} H_{2}\right), 68.5 \& 68.6$ (C6), 73.5\&73.6 $\left(\mathrm{PhCH}_{2} \mathrm{O}\right), 75.1$ (C5), 75.1\&75.2 $\left(\mathrm{PhCH}_{2} \mathrm{O}\right), 75.5 \& 76.6\left(\mathrm{PhCH}_{2} \mathrm{O}\right), 76.4 \& 77.1(\mathrm{C} \gamma), 77.5$ (C4), 82.8\&82.9 (C3), 101.1 (C1), $118.9 \& 119.0\left(\mathrm{CH}=\mathrm{CH}_{2}\right), 119.9,125.0,125.1,125.2,127.0,127.6,127.7,127.8,127.9,128.0$, $128.3,128.4,131.5 \& 131.7 \quad\left(\underline{\mathrm{CH}}=\mathrm{CH}_{2}\right), 137.7,137.9,141.2,143.6,143.7,143.9,144.1$, 
154.3\&154.8 (Fmoc $\underline{\mathrm{C}}=\mathrm{O}), 172.1 \& 172.2$ ( $\underline{\mathrm{COOR}})$. HRMS (FAB+, NBA) calcd for $\mathrm{MH}^{+}$ $\mathrm{C}_{50} \mathrm{H}_{51} \mathrm{~N}_{4} \mathrm{O}_{9} 851.3656$, obsd 851.3677.

\section{4-O-(2-acetamido-3,4,6-tri- $O$-benzyl-2-deoxy- $\beta$-D-glucopyranosyl)- $N$ -}

fluorenylmethoxycarbonyl-trans-4-hydroxy-L-proline allyl ester (17)

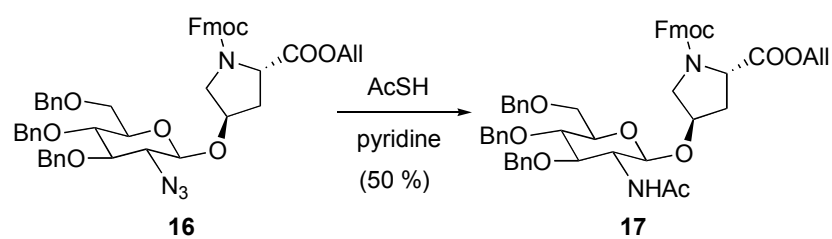

Thiolacetic acid $(217 \mu \mathrm{L}, 232 \mathrm{mg}, 3.06 \mathrm{mmol}, 40.0$ equiv.) was added to a solution of 4-O-(2azido-3,4,6-tri- $O$-benzyl-2-deoxy- $\beta$-D-glucopyranosyl)- $N$-fluorenylmethoxycarbonyl-trans-4hydroxy-L-proline allyl ester (16) (65 mg, $0.077 \mathrm{mmol}, 1.0$ equiv.) in dry pyridine ( $2 \mathrm{~mL})$ and the solution was stirred at RT under $\mathrm{N}_{2}$ for $48 \mathrm{~h}$. The mixture was concentrated and azeotroped twice from toluene. The residue was taken up in $\mathrm{CH}_{2} \mathrm{Cl}_{2}(100 \mathrm{~mL})$ and washed with a 1:1 solution of $\mathrm{NaHCO}_{3}$-brine $(100 \mathrm{~mL})$. The aqueous layer was extracted again with $\mathrm{CH}_{2} \mathrm{Cl}_{2}(100 \mathrm{~mL})$. The combined organic layers were dried over $\mathrm{MgSO}_{4}$, filtered, and concentrated. The residue was purified by flash column chromatography, eluting with 1:1 to 2:1 EtOAc-hexanes, to give $\mathbf{1 7}$ as a colorless solid (33 mg, $50 \%) . \quad R_{f} 0.32$ (2:1 EtOAc-hexanes). $[\alpha]_{\mathrm{D}}{ }^{20}=-19.1^{\circ}\left(c 1.0, \mathrm{CHCl}_{3}\right) .{ }^{1} \mathrm{H}$ $\operatorname{NMR}\left(500 \mathrm{MHz}, \mathrm{CDCl}_{3}\right) \delta 1.82 \& 1.84\left(\mathrm{~s}, 3 \mathrm{H}, \mathrm{NHCOC}_{3}\right), 2.01-2.09(\mathrm{~m}, 1 \mathrm{H}, \mathrm{H} \beta$ '), $2.35-2.40(\mathrm{~m}$,

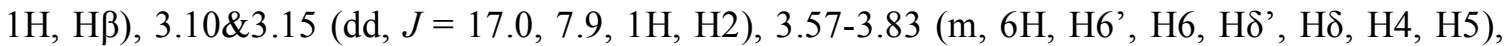
4.12-4.68 (m, 12H, H3, H $\gamma, \mathrm{H} \alpha, 2$ x $\mathrm{PhCH}_{2} \mathrm{O}, \mathrm{CH}_{2} \mathrm{CH}=\mathrm{CH}_{2}$, Fmoc- $\mathrm{CH}_{2}$, Fmoc-CH), 4.76-4.84 $\left(\mathrm{m}, 2 \mathrm{H}, \mathrm{PhCH}_{2} \mathrm{O}\right), 5.05 \& 5.13(\mathrm{~d}, J=8.0 \mathrm{~Hz}, 1 \mathrm{H}, \mathrm{H} 1), 5.17-5.34\left(\mathrm{~m}, 2 \mathrm{H}, \mathrm{CH}=\mathrm{C}_{2}\right), 5.59 \& 5.62$ $\left(\mathrm{d}, J=7.1 \mathrm{~Hz}, 1 \mathrm{H}, \mathrm{N} \underline{\mathrm{HCOCH}}{ }_{3}\right), 5.78-5.94\left(\mathrm{~m}, 1 \mathrm{H}, \mathrm{C} \underline{\mathrm{H}}=\mathrm{CH}_{2}\right), 7.18-7.40$ (m, 19H, Ar- $\underline{\mathrm{H}} \mathrm{x} 4, \mathrm{Ph}-$ $\underline{\mathrm{H}}), 7.53-7.60(\mathrm{~m}, 2 \mathrm{H}, \mathrm{Ar}-\underline{\mathrm{H}}), 7.75(\mathrm{t}, J=7.1 \mathrm{~Hz}, 2 \mathrm{H}, \mathrm{Ar}-\underline{\mathrm{H}}) ;{ }^{13} \mathrm{C} \mathrm{NMR}\left(125 \mathrm{MHz}, \mathrm{CDCl}_{3}\right) \delta 23.5$ $\left(\mathrm{NHCOCH}_{3}\right), \quad 35.4 \& 36.6(\mathrm{C} \beta), 47.1 \& 47.2$ (Fmoc- $\left.\mathrm{CH}\right), 52.9 \& 53.3$ (C反), 57.5\&57.7 (C2), $57.9 \& 58.2(\mathrm{C \alpha}), 65.8\left(\underline{\mathrm{CH}}_{2} \mathrm{CH}=\mathrm{CH}_{2}\right), 67.5 \& 67.5\left(\right.$ Fmoc- $\left.\underline{\mathrm{CH}}_{2}\right), 68.8(\mathrm{C} 6), 73.5\left(\mathrm{PhCH}_{2} \mathrm{O}\right)$, $74.6 \& 74.8\left(\mathrm{PhCH}_{2} \mathrm{O}\right), 74.6 \& 74.9$ (C5), $74.9 \& 76.0\left(\mathrm{PhCH}_{2} \mathrm{O}\right), 75.3 \& 76.1(\mathrm{C} \gamma), 78.6 \& 78.8(\mathrm{C} 4)$, 79.8 (C3), $97.7 \& 98.1(\mathrm{C} 1), 118.6 \& 118.9\left(\mathrm{CH}=\underline{\mathrm{C}} \mathrm{H}_{2}\right), 119.9,124.9,125.1,125.2,127.0,127.1$, $127.7,127.8,128.3,128.4,128.5,131.4 \& 131.6\left(\underline{\mathrm{CH}}=\mathrm{CH}_{2}\right), 137.9,138.0,138.3,141.2,143.8$, 143.9, 144.2, $154.3 \& 154.8($ Fmoc $\underline{\mathrm{C}}=\mathrm{O}), 170.3\left(\mathrm{NH}^{\mathrm{COCH}}{ }_{3}\right), 171.9$ (ㅁOOR). HRMS (FAB+ NBA) calcd for $\mathrm{MH}^{+} \mathrm{C}_{52} \mathrm{H}_{54} \mathrm{~N}_{2} \mathrm{O}_{10} 867.3857$, obsd 867.3872. 


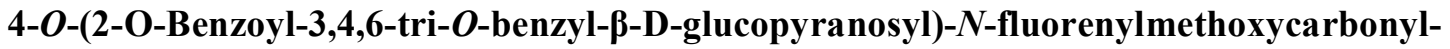
trans-4-hydroxy-L-proline allyl ester (18)

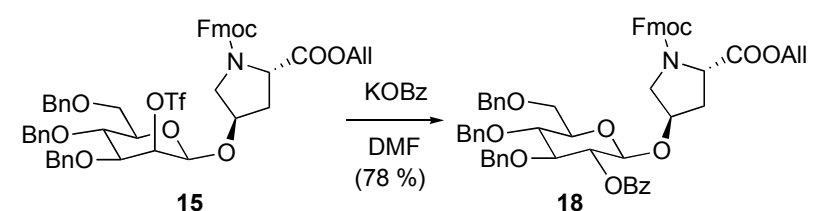

Potassium benzoate ( $31 \mathrm{mg}, 0.196 \mathrm{mmol}, 2.0$ equiv.) was added to a solution of 4-O-(3,4,6-tri- $O$ benzyl-2-O-trifluoromethanesulfonyl- $\beta$-D-mannopyranosyl)- $N$-fluorenylmethoxycarbonyl-trans4-hydroxy-L-proline allyl ester (15) (94 mg, $0.098 \mathrm{mmol}, 1.0$ equiv.) in dry DMF (2 mL) and the solution was stirred at RT under $\mathrm{N}_{2}$ for $4 \mathrm{~h}$. The reaction was diluted with $\mathrm{CH}_{2} \mathrm{Cl}_{2}(100 \mathrm{~mL})$, washed with water $(2 \times 100 \mathrm{~mL})$, dried over $\mathrm{MgSO}_{4}$, filtered, and concentrated. The residue was purified by flash column chromatography, eluting with 2:1 hexanes-EtOAc, to give $\mathbf{1 8}$ as a colorless oil (71 mg, $78 \%) . \quad R_{f} 0.70$ (2:1 EtOAc-hexanes). $[\alpha]_{\mathrm{D}}{ }^{20}=-15.5^{\circ}\left(c 1.0, \mathrm{CHCl}_{3}\right) .{ }^{1} \mathrm{H}$

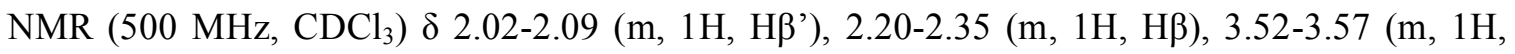

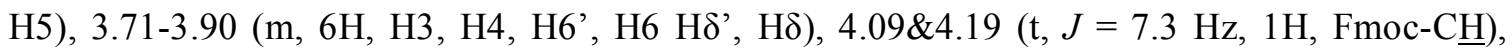
4.24-4.67 (m, 11H, H1, H $\gamma, \mathrm{H} \alpha, 2$ x PhC $\underline{\mathrm{H}}_{2} \mathrm{O}, \mathrm{C}_{2} \mathrm{CH}=\mathrm{CH}_{2}, \mathrm{Fmoc}_{-} \mathrm{CH}_{2}$ ), 4.73-4.83 (m, 2H, $\left.\mathrm{PhCH}_{2} \mathrm{O}\right), 5.13-5.28\left(\mathrm{~m}, 3 \mathrm{H}, \mathrm{H} 2, \mathrm{CH}=\mathrm{CH}_{2}\right), 5.73-5.88\left(\mathrm{~m}, 1 \mathrm{H}, \mathrm{C} \underline{\mathrm{H}}=\mathrm{CH}_{2}\right), 7.12-7.58(\mathrm{~m}, 24 \mathrm{H}, \mathrm{Ar}-$ $\underline{\mathrm{H}}), 7.74(\mathrm{t}, J=7.8 \mathrm{~Hz}, 2 \mathrm{H}, \mathrm{Ar}-\underline{\mathrm{H}}), 7.99-8.01(\mathrm{~m}, 2 \mathrm{H}, \mathrm{Ar}-\underline{\mathrm{H}}) ;{ }^{13} \mathrm{C} \mathrm{NMR}\left(125 \mathrm{MHz}, \mathrm{CDCl}_{3}\right) \delta$ $35.5 \& 37.0(\mathrm{C} \beta), 47.1 \& 47.2(\mathrm{Fmoc}-\underline{\mathrm{CH}}), 52.5 \& 53.1(\mathrm{C} \delta), 57.3 \& 57.6(\mathrm{C} \alpha), 65.7\left(\underline{\mathrm{CH}_{2}} \mathrm{CH}=\mathrm{CH}_{2}\right)$, $67.7\left(\right.$ Fmoc- $\left.\underline{\mathrm{H}}_{2}\right), 68.7 \& 68.6(\mathrm{C} 6), 73.5\left(\mathrm{PhCH}_{2} \mathrm{O}\right), 73.6(\mathrm{C} 2), 75.0\left(\mathrm{Ph}^{\mathrm{C}} \mathrm{H}_{2} \mathrm{O}\right), 75.2\left(\mathrm{PhCH}_{2} \mathrm{O}\right)$, $75.4 \& 75.5$ (C5), 76.5\&76.6 (C $\gamma), 77.8$ (C4), 82.6 (C3), 100.2\&100.6 (C1), 118.9\&119.0 $\left(\mathrm{CH}=\underline{\mathrm{CH}}_{2}\right), 119.8,119.9,125.1,125.2,127.1,127.6,127.7,127.8,127.9,128.0,128.2,128.4$, $128.5,131.5 \& 131.7\left(\underline{\mathrm{CH}}=\mathrm{CH}_{2}\right), 133.2,137.7,137.8,137.9,138.0,141.2,143.9,144.1,165.0$ (Fmoc $\underline{\mathrm{C}}=\mathrm{O}), 172.0$ ( $\underline{\mathrm{COOR}}) .172 .1 \quad(\mathrm{Bz} \underline{\mathrm{C}}=\mathrm{O}) . \quad \mathrm{HRMS}(\mathrm{FAB}+, \mathrm{NBA})$ calcd for $\mathrm{MH}^{+}$ $\mathrm{C}_{57} \mathrm{H}_{55} \mathrm{NO}_{11}$ 930.3853, obsd 930.3865 . 
${ }^{1} \mathrm{H}$ NMR spectrum of 3,4,6-tri- $O$-benzyl- $D$-mannopyranose (7)

$\mathrm{CDCl}_{3}, 500 \mathrm{MHz}$

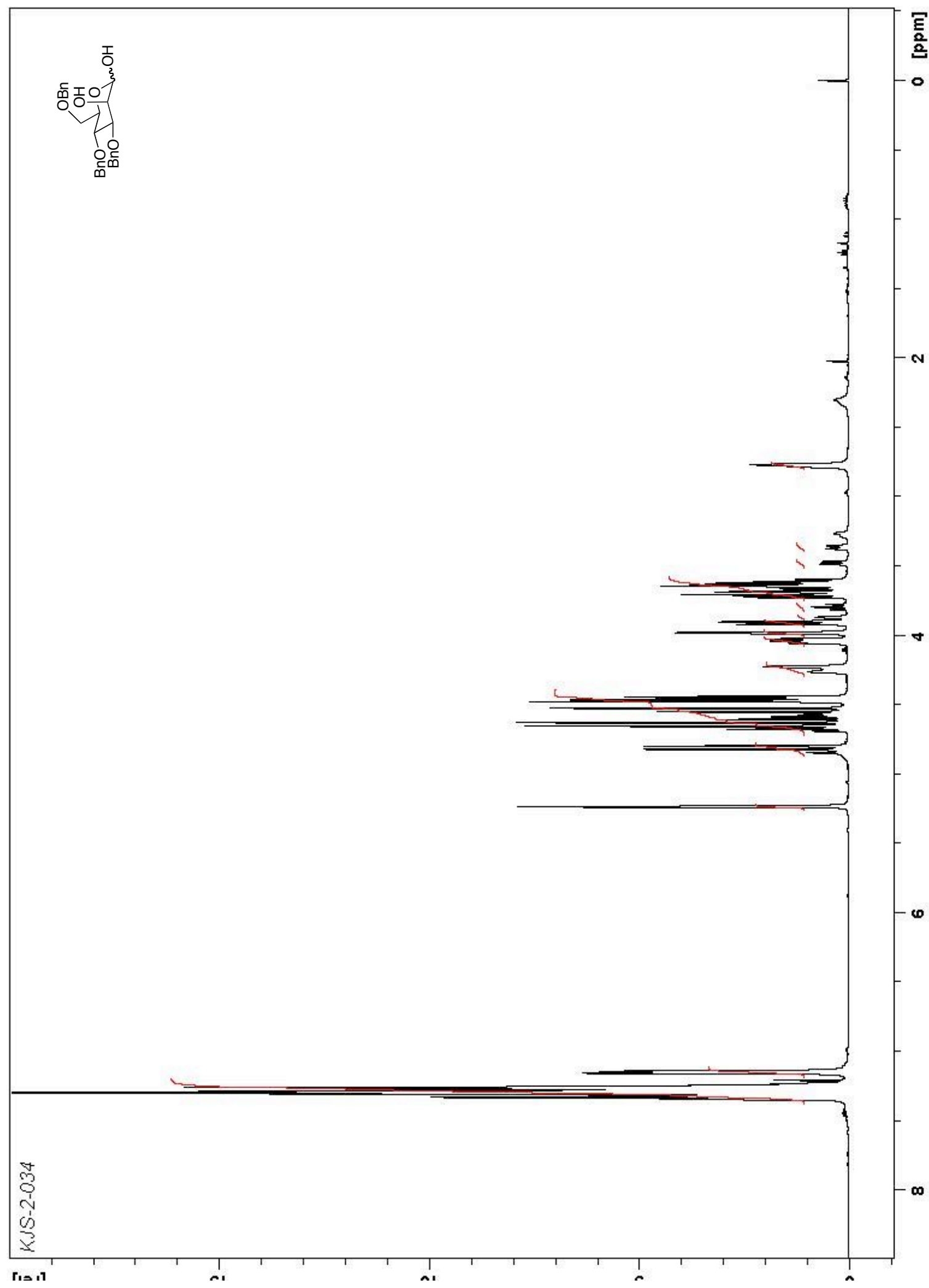


${ }^{13} \mathrm{C}$ NMR spectrum of 3,4,6-tri-O-benzyl-D-mannopyranose (7)

$\mathrm{CDCl}_{3}, 125 \mathrm{MHz}$

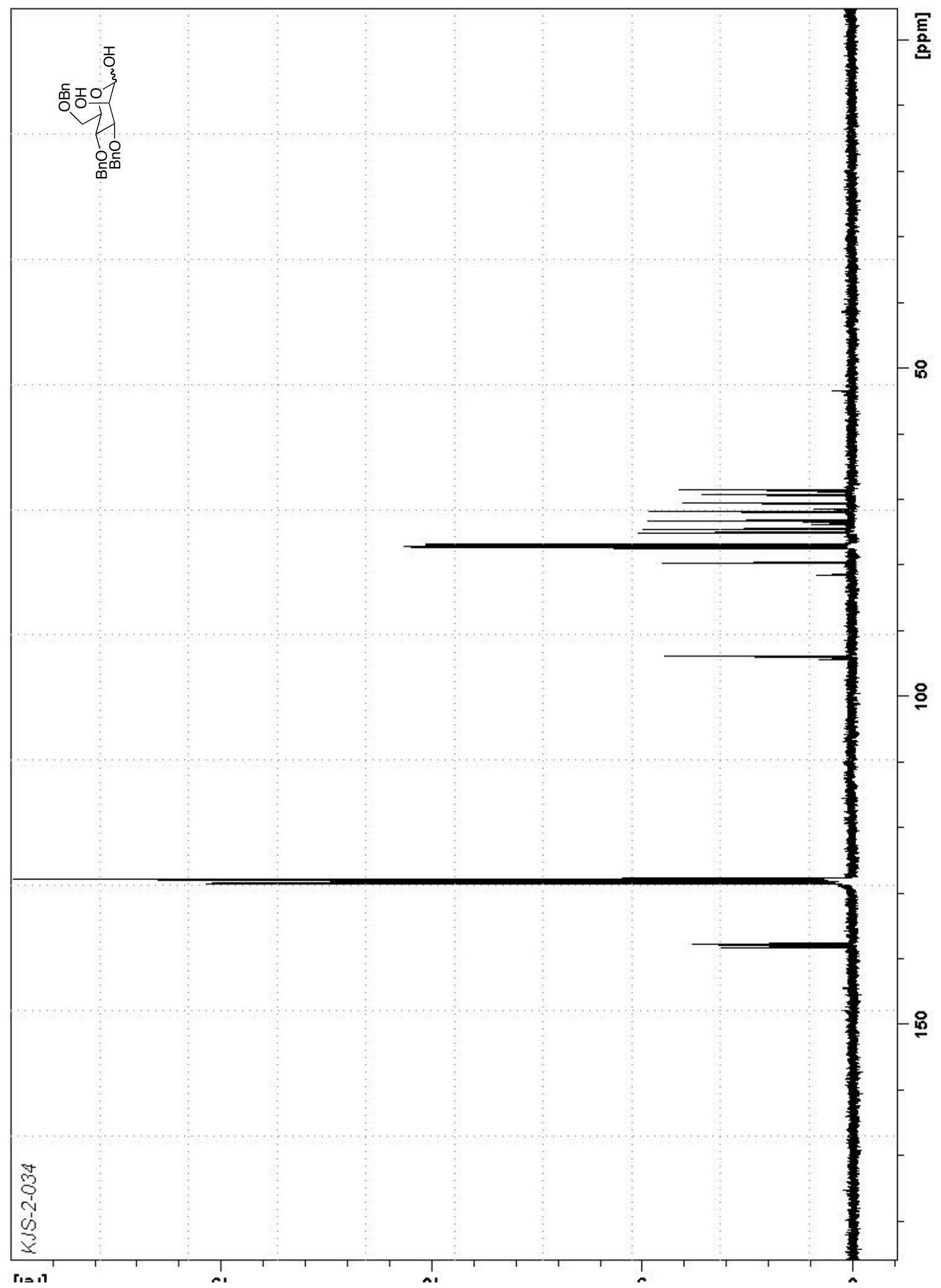


${ }^{1} \mathrm{H}$ NMR spectrum of $N \alpha$-fluorenylmethoxycarbonyl-trans-4-hydroxy-L-proline allyl ester (trans-8) $\mathrm{CDCl}_{3}, 500 \mathrm{MHz}$

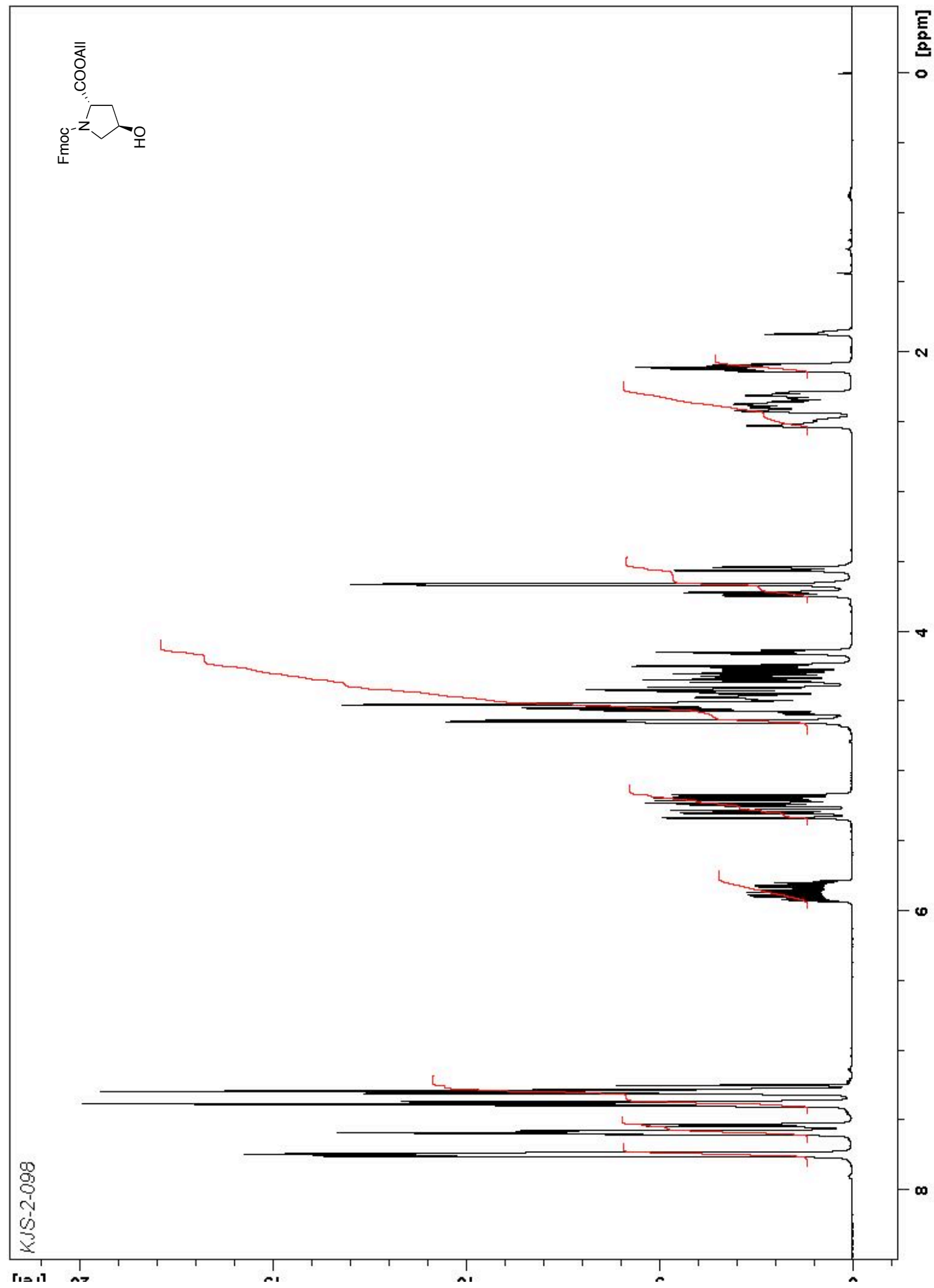


${ }^{13} \mathrm{C}$ NMR spectrum of $N \alpha$-fluorenylmethoxycarbonyl-trans-4-hydroxy-L-proline allyl ester (trans-8) $\mathrm{CDCl}_{3}, 125 \mathrm{MHz}$

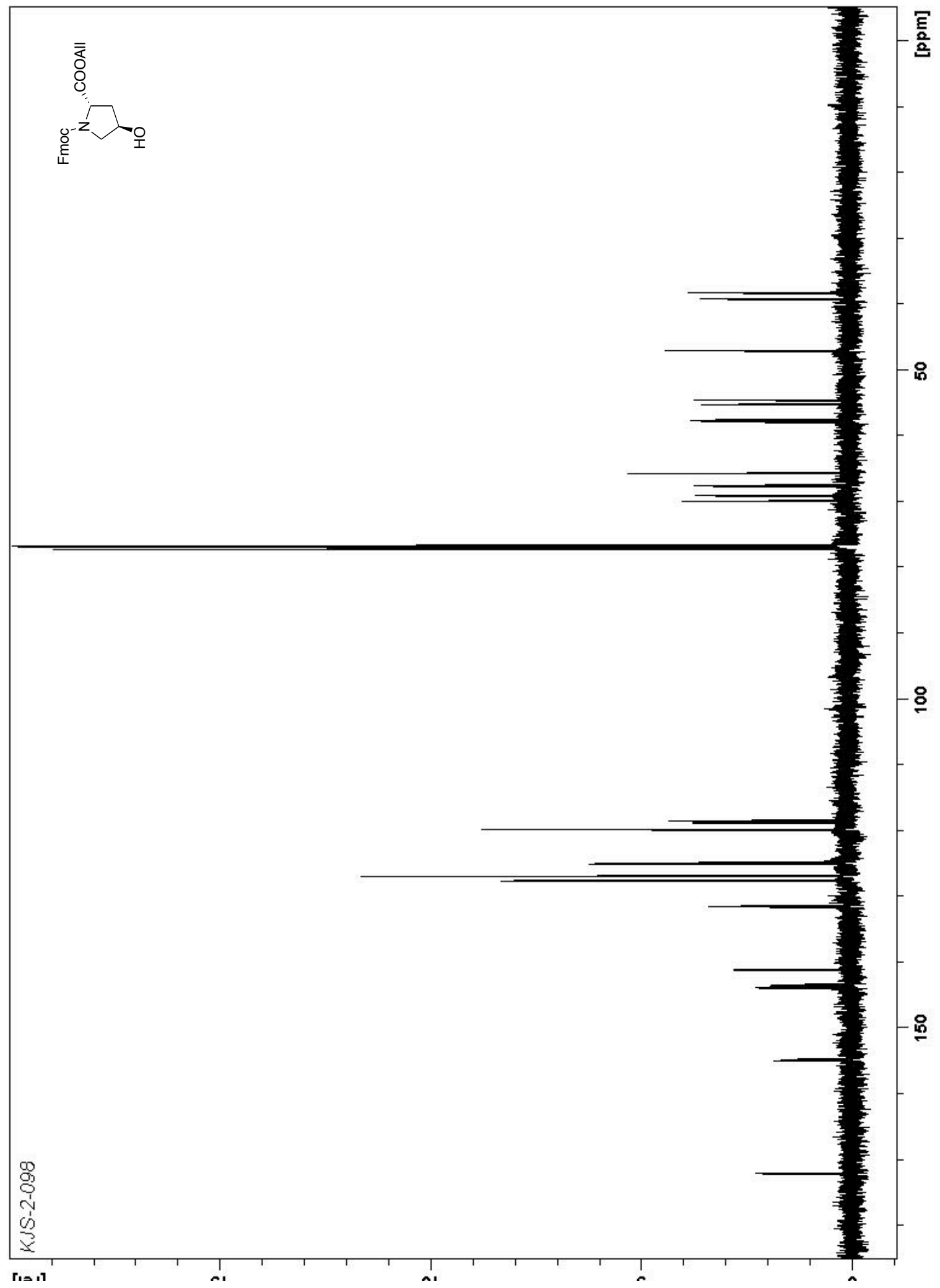


${ }^{1} \mathrm{H}$ NMR spectrum of $N \alpha$-fluorenylmethoxycarbonyl-trans-4-trifluoromethanesulfonoyloxy-L-proline allyl ester (trans-5), $\mathrm{CDCl}_{3}, 500 \mathrm{MHz}$

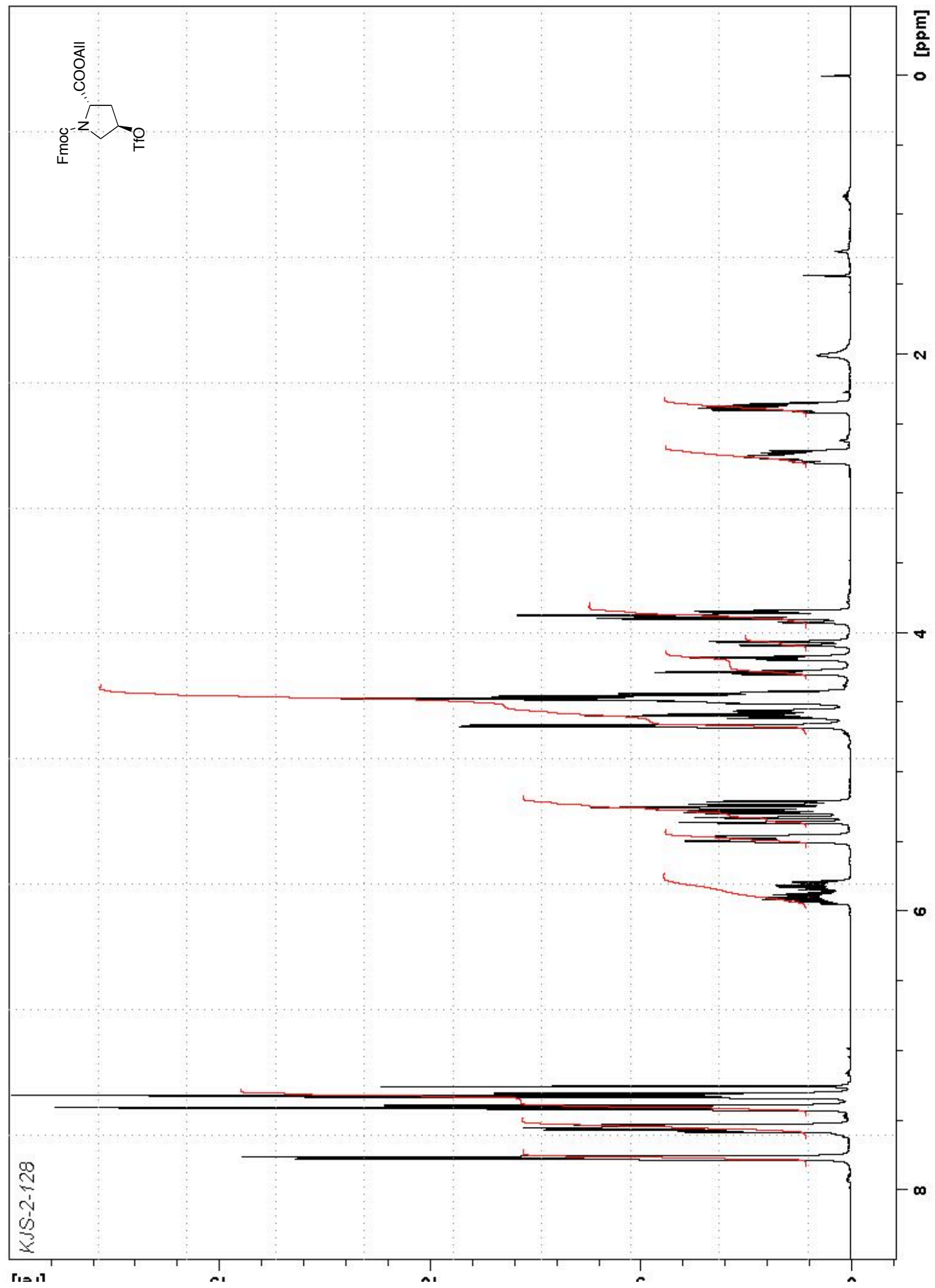


${ }^{13} \mathrm{C}$ NMR spectrum of $N \alpha$-fluorenylmethoxycarbonyl-trans-4-trifluoromethanesulfonoyloxy-Lproline allyl ester (trans-5), $\mathrm{CDCl}_{3}, 125 \mathrm{MHz}$

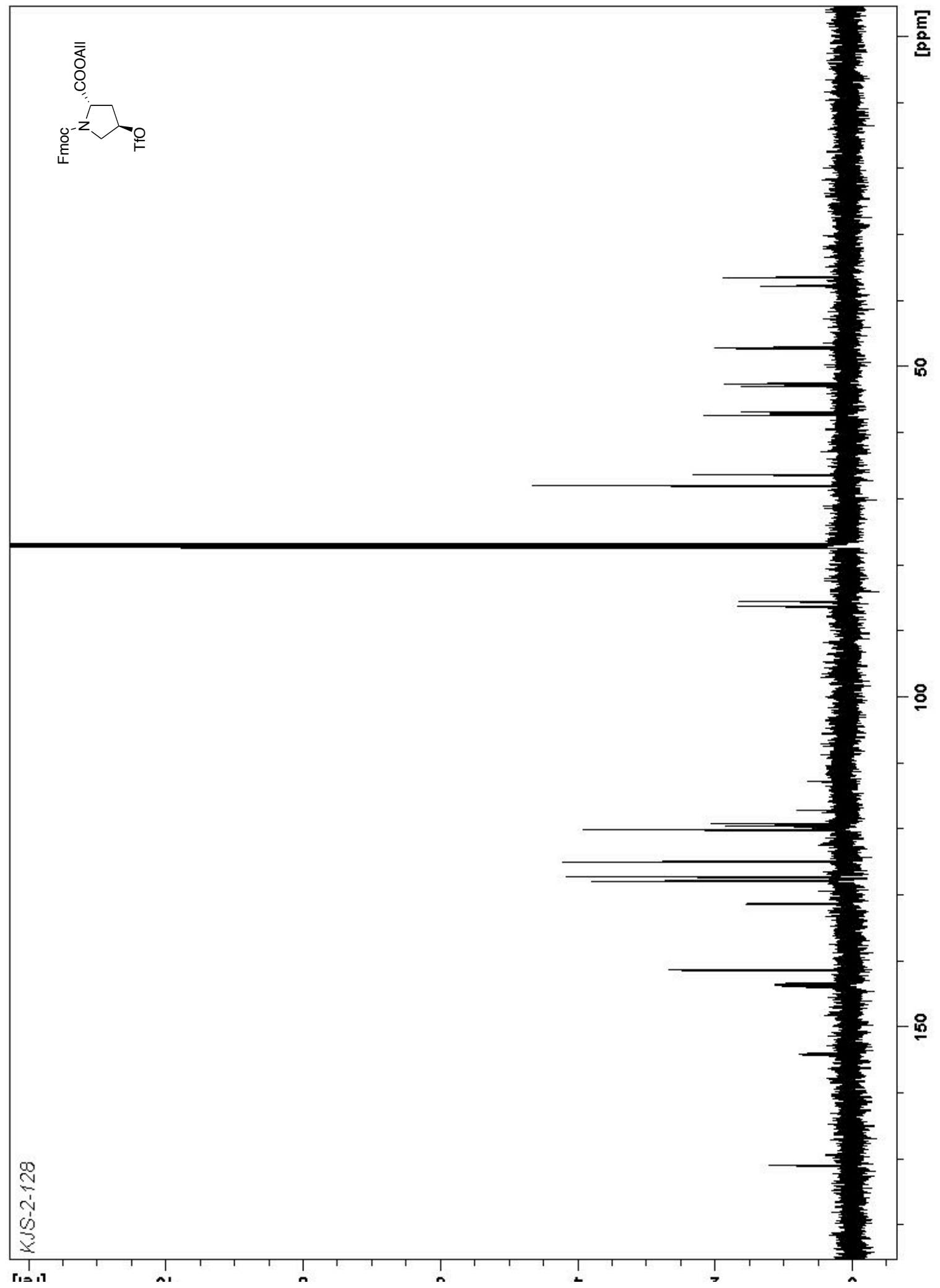


${ }^{1} \mathrm{H}$ NMR spectrum of $\mathrm{N} \alpha$-fluorenylmethoxycarbonyl-cis-4-p-nitrobenzoyloxy-L-proline allyl ester (cis-9), $\mathrm{CDCl}_{3}, 500 \mathrm{MHz}$

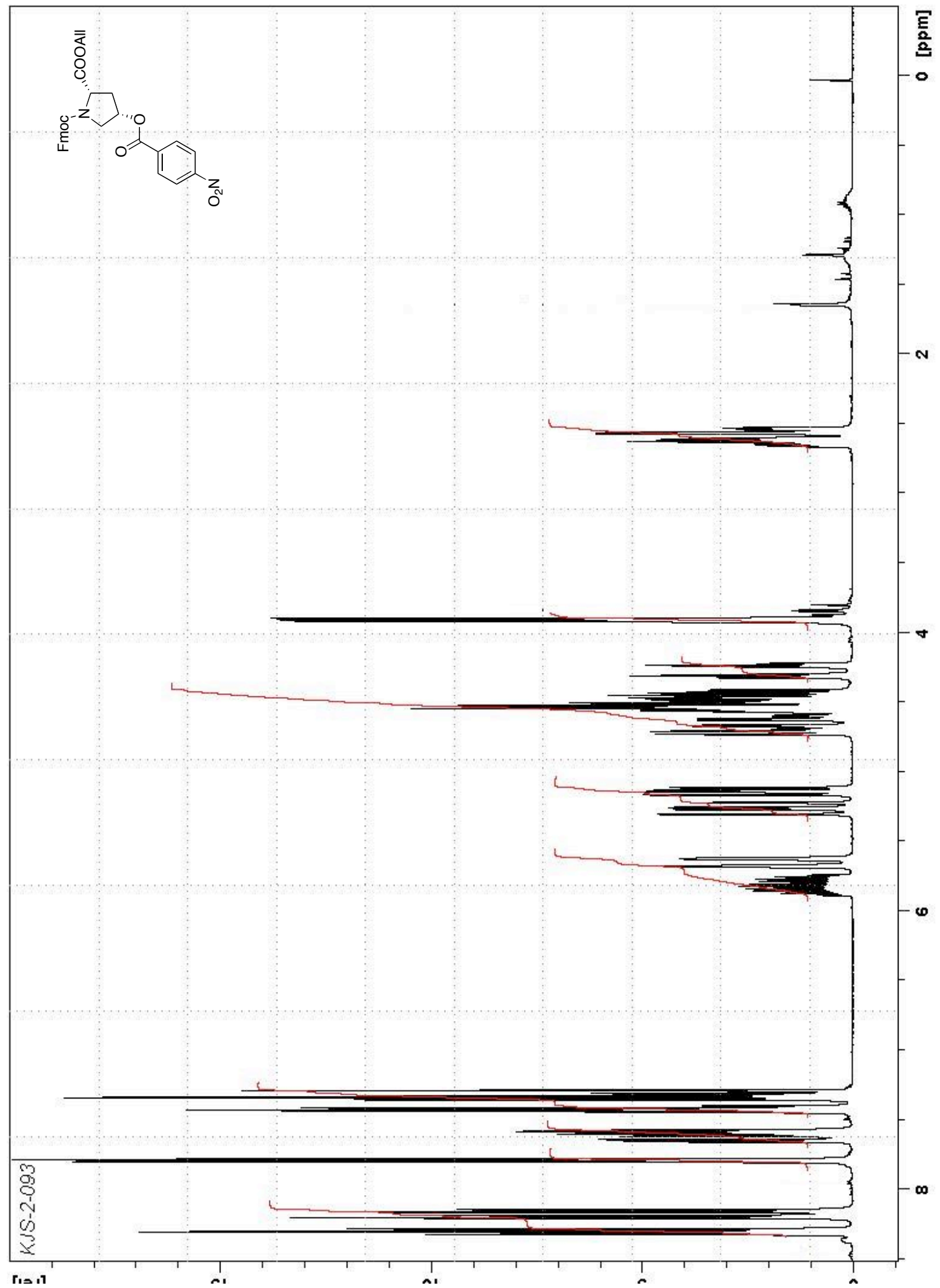




$$
7
$$


${ }^{1} \mathrm{H}$ NMR spectrum of $\mathrm{N} \alpha$-fluorenylmethoxycarbonyl-cis-4-hydroxy-L-proline allyl ester (cis-8) $\mathrm{CDCl}_{3}, 500 \mathrm{MHz}$

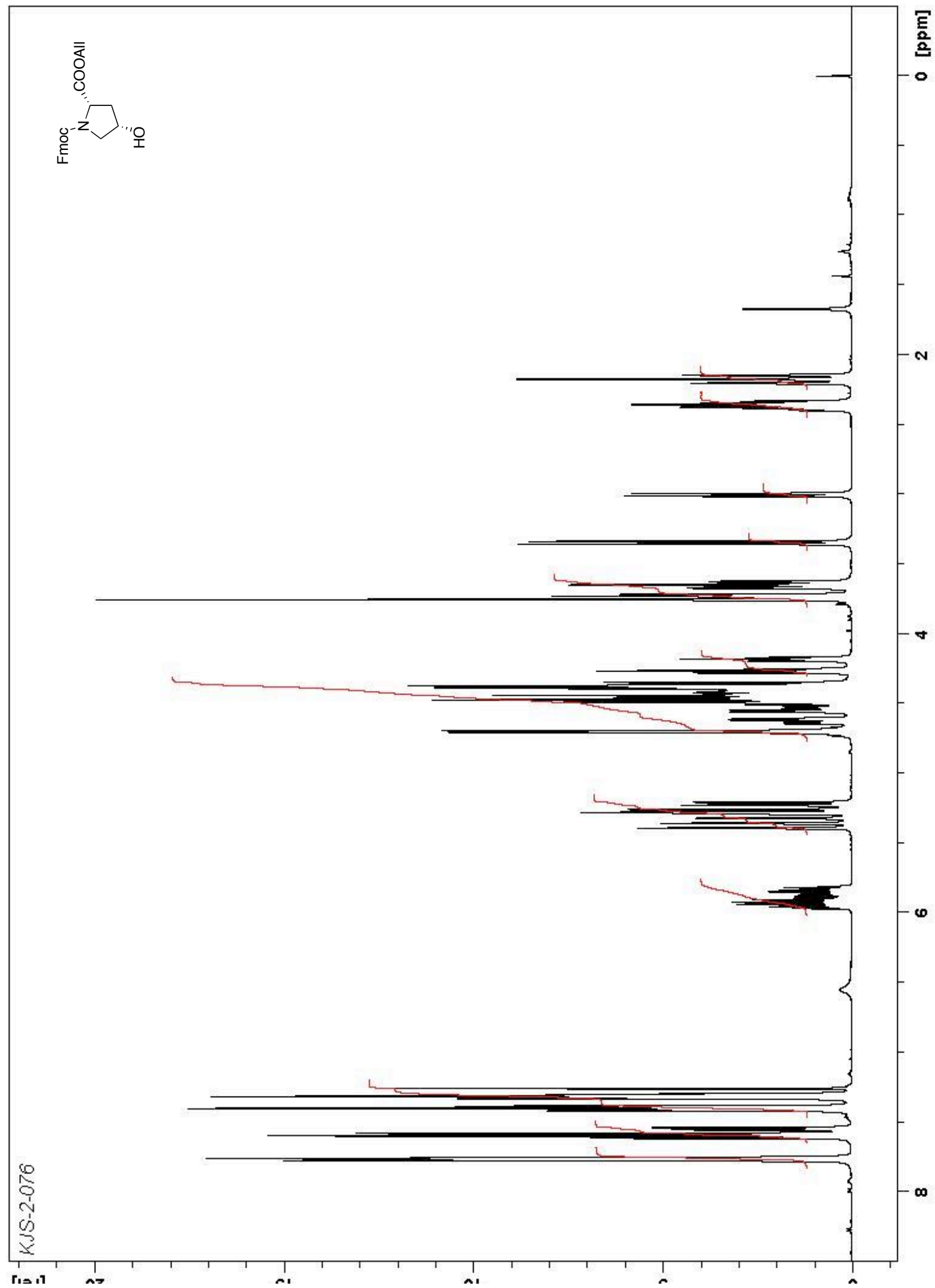


${ }^{13} \mathrm{C}$ NMR spectrum of $N \alpha$-fluorenylmethoxycarbonyl-cis-4-hydroxy-L-proline allyl ester (cis-8) $\mathrm{CDCl}_{3}, 125 \mathrm{MHz}$

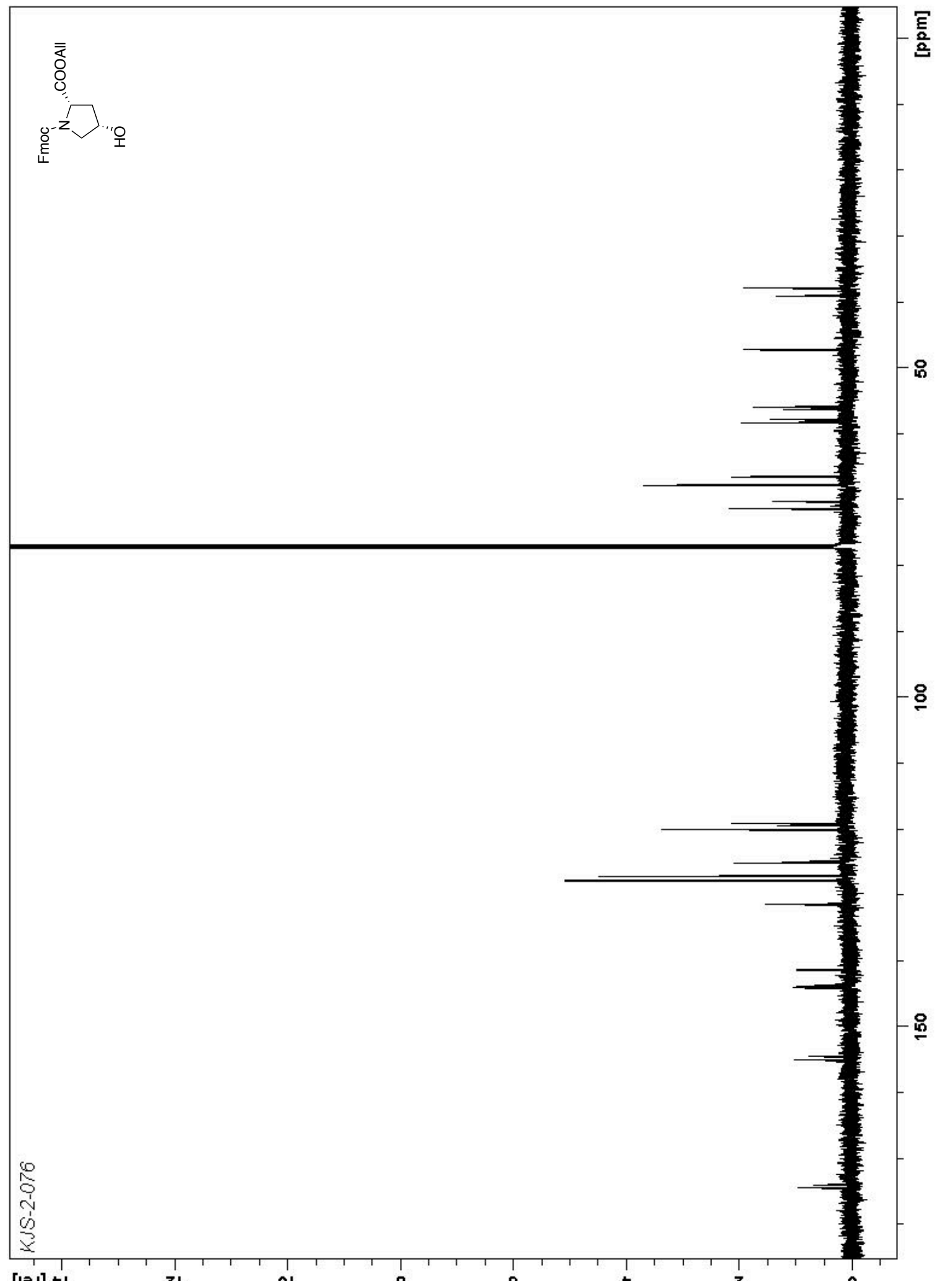


${ }^{1} \mathrm{H}$ NMR spectrum of $\mathrm{N} \alpha$-fluorenylmethoxycarbonyl-cis-4-trifluormethanesulfonyloxy-L-proline allyl ester (cis-5), $\mathrm{CDCl}_{3}, 125 \mathrm{MHz}$

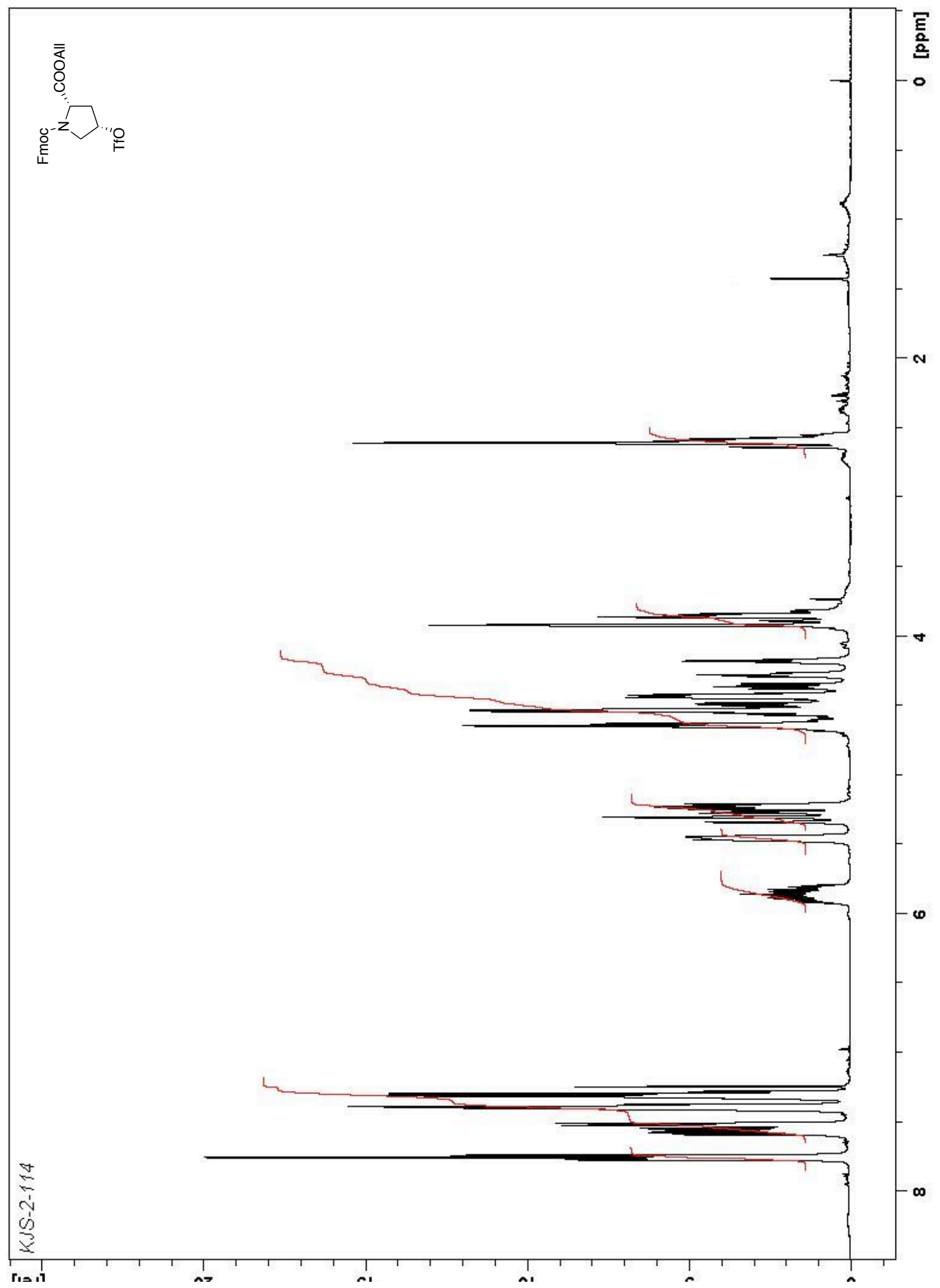


${ }^{13} \mathrm{C}$ NMR spectrum of $N \alpha$-fluorenylmethoxycarbonyl-cis-4-trifluormethanesulfonyloxy-L-proline allyl ester (cis-5), $\mathrm{CDCl}_{3}, 125 \mathrm{MHz}$

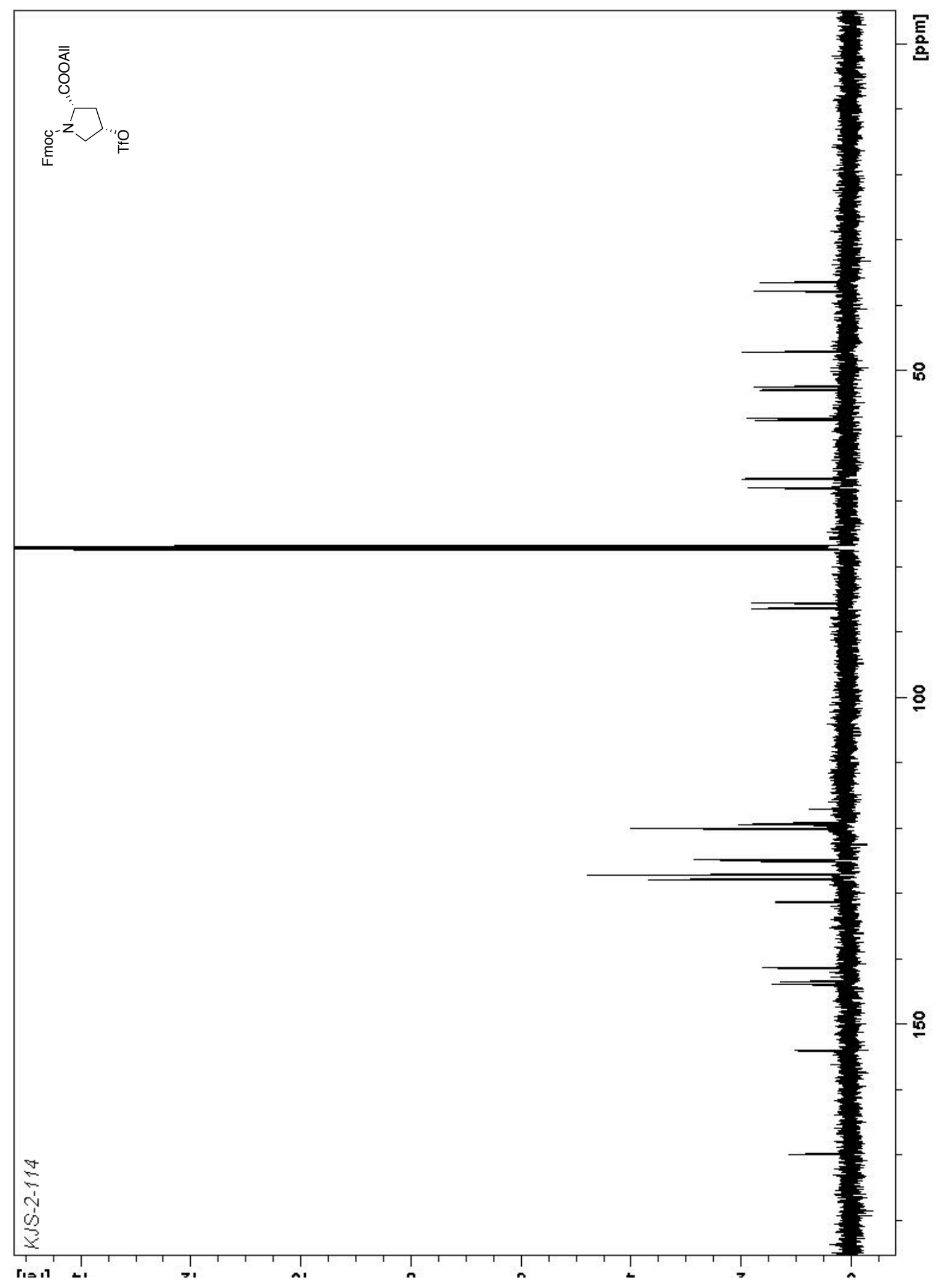


${ }^{1} \mathrm{H}$ NMR spectrum of 4-O-(3,4,6-tri-O-benzyl- $\beta$ - $D$-mannopyranosyl)- $N \alpha$-fluorenylmethoxycarbonyl-trans-4-hydroxy- $L$-proline allyl ester (trans-4), $\mathrm{CDCl}_{3}, 500 \mathrm{MHz}$

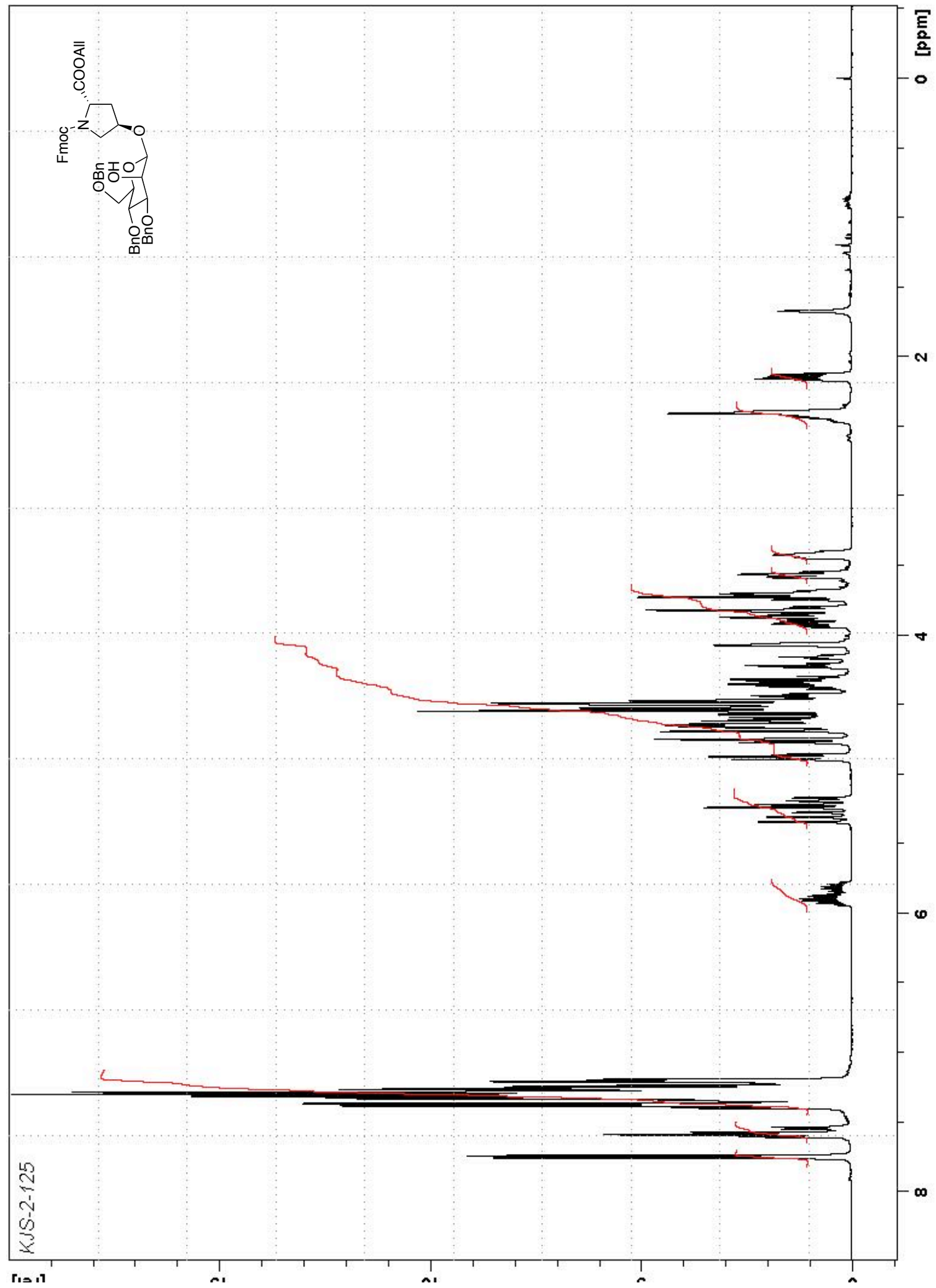


${ }^{13} \mathrm{C}$ NMR spectrum of 4-O-(3,4,6-tri- $O$-benzyl- $\beta$ - $D$-mannopyranosyl)- $N \alpha$-fluorenylmethoxycarbonyl-trans-4-hydroxy- $L$-proline allyl ester (trans-4), $\mathrm{CDCl}_{3}, 125 \mathrm{MHz}$

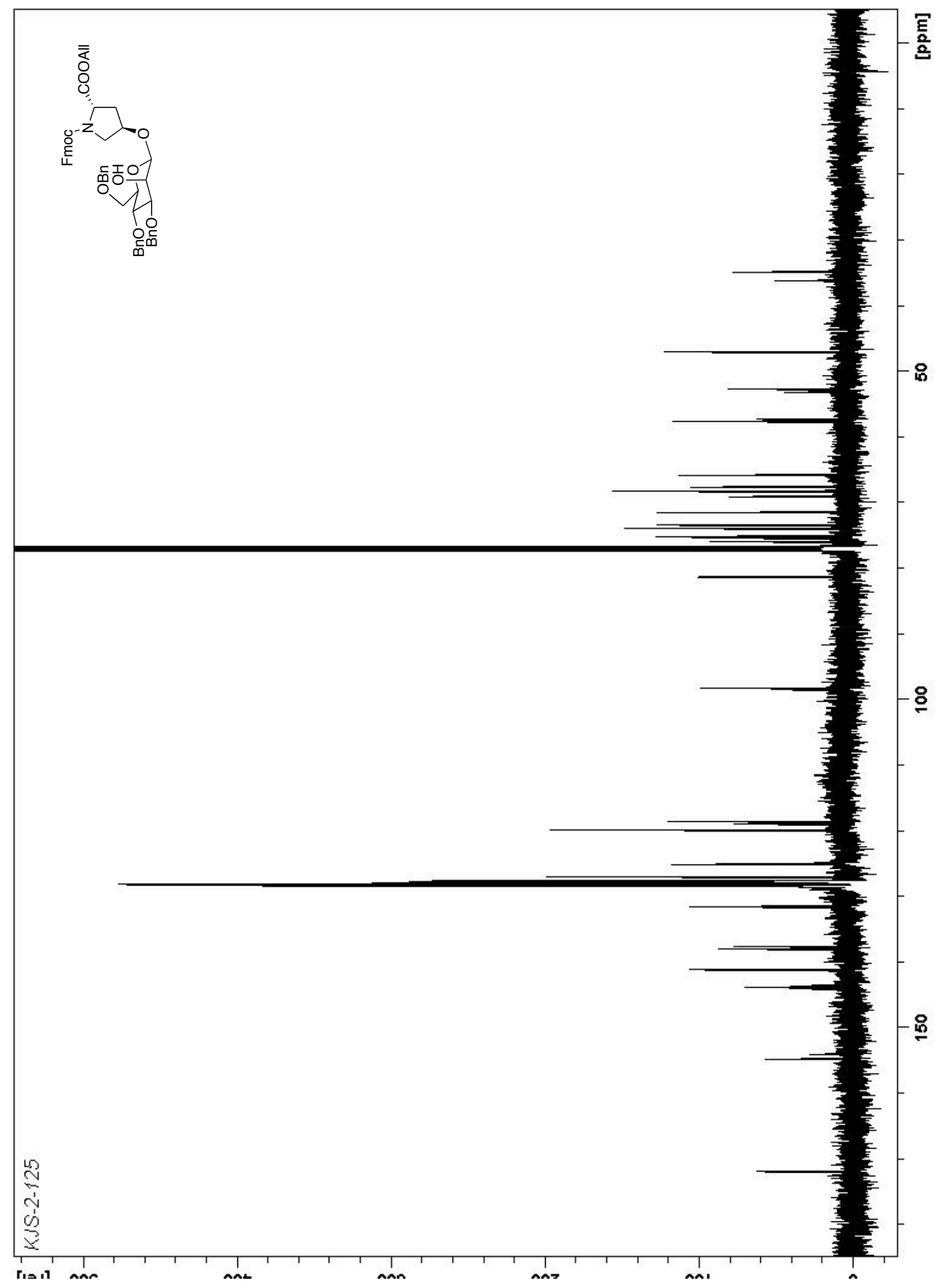


${ }^{1} \mathrm{H}$ NMR spectrum of 4-O-(3,4,6-tri-O-benzyl- $\beta$ - $D$-mannopyranosyl)- $N \alpha$-fluorenylmethoxycarbonyl-cis-4-hydroxy- $L$-proline allyl ester (cis-4), $\mathrm{CDCl}_{3}, 500 \mathrm{MHz}$

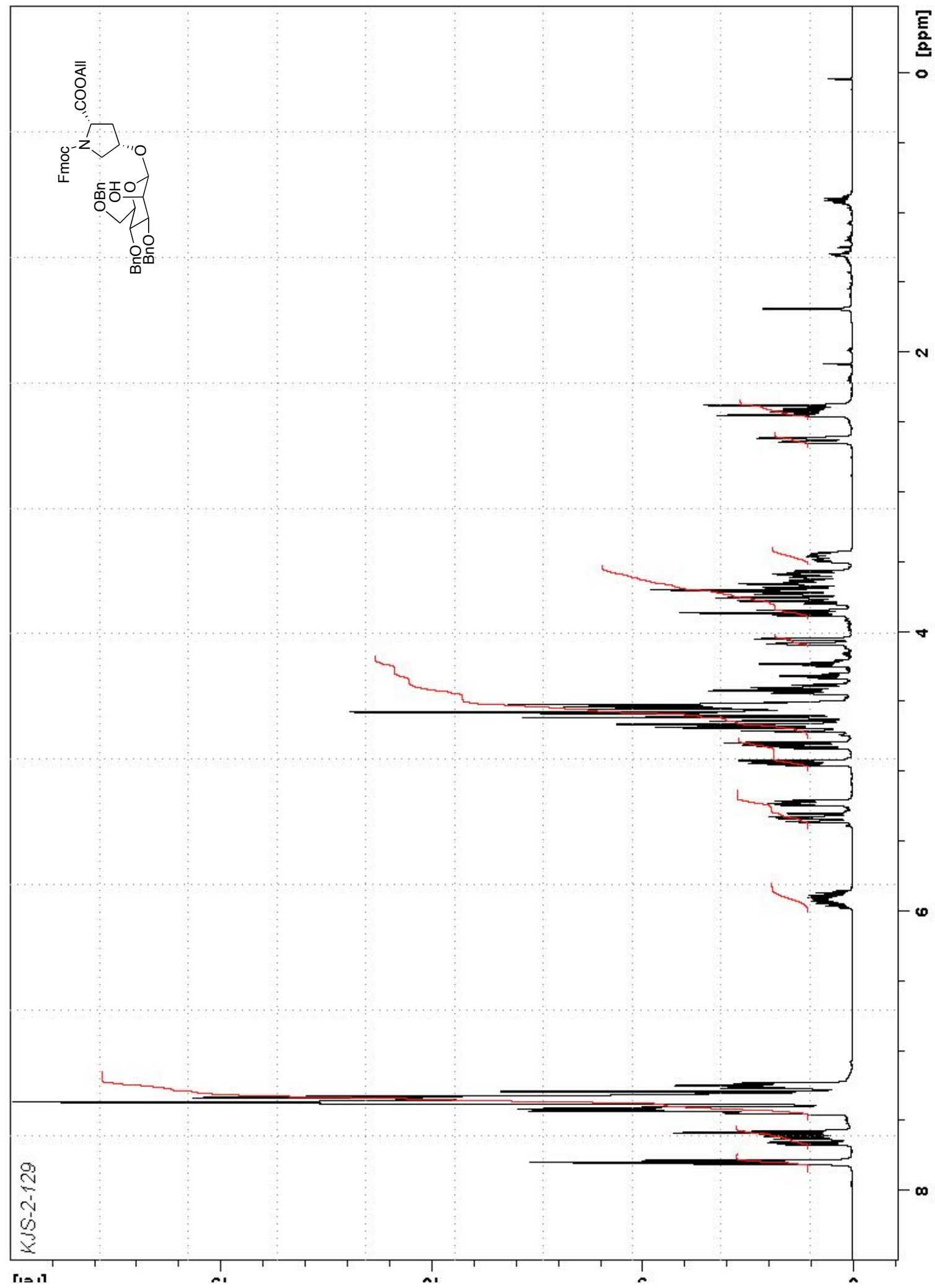


${ }^{13} \mathrm{C}$ NMR spectrum of 4-O-(3,4,6-tri-O-benzyl- $\beta$ - $D$-mannopyranosyl)- $N \alpha$-fluorenylmethoxycarbonyl-cis-4-hydroxy- L-proline allyl ester (cis-4), $\mathrm{CDCl}_{3}, 125 \mathrm{MHz}$

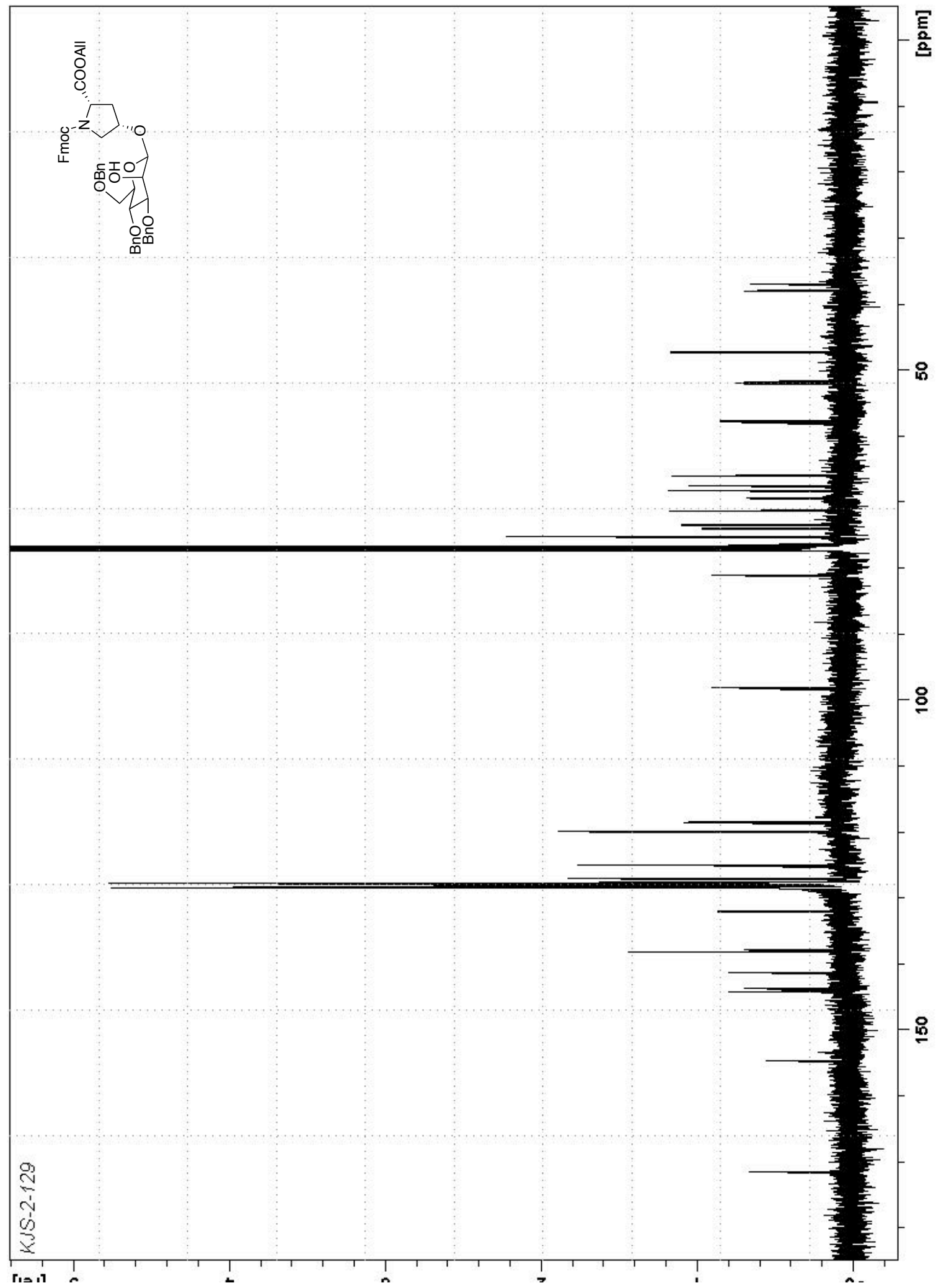


${ }^{1} \mathrm{H}$ NMR spectrum of 4-O-(3,4,6-tri-O-benzyl- $\beta$ - $D$-mannopyranosyl)-trans-4-hydroxy-L-proline allyl ester (trans-14), $\mathrm{CDCl}_{3}, 500 \mathrm{MHz}$

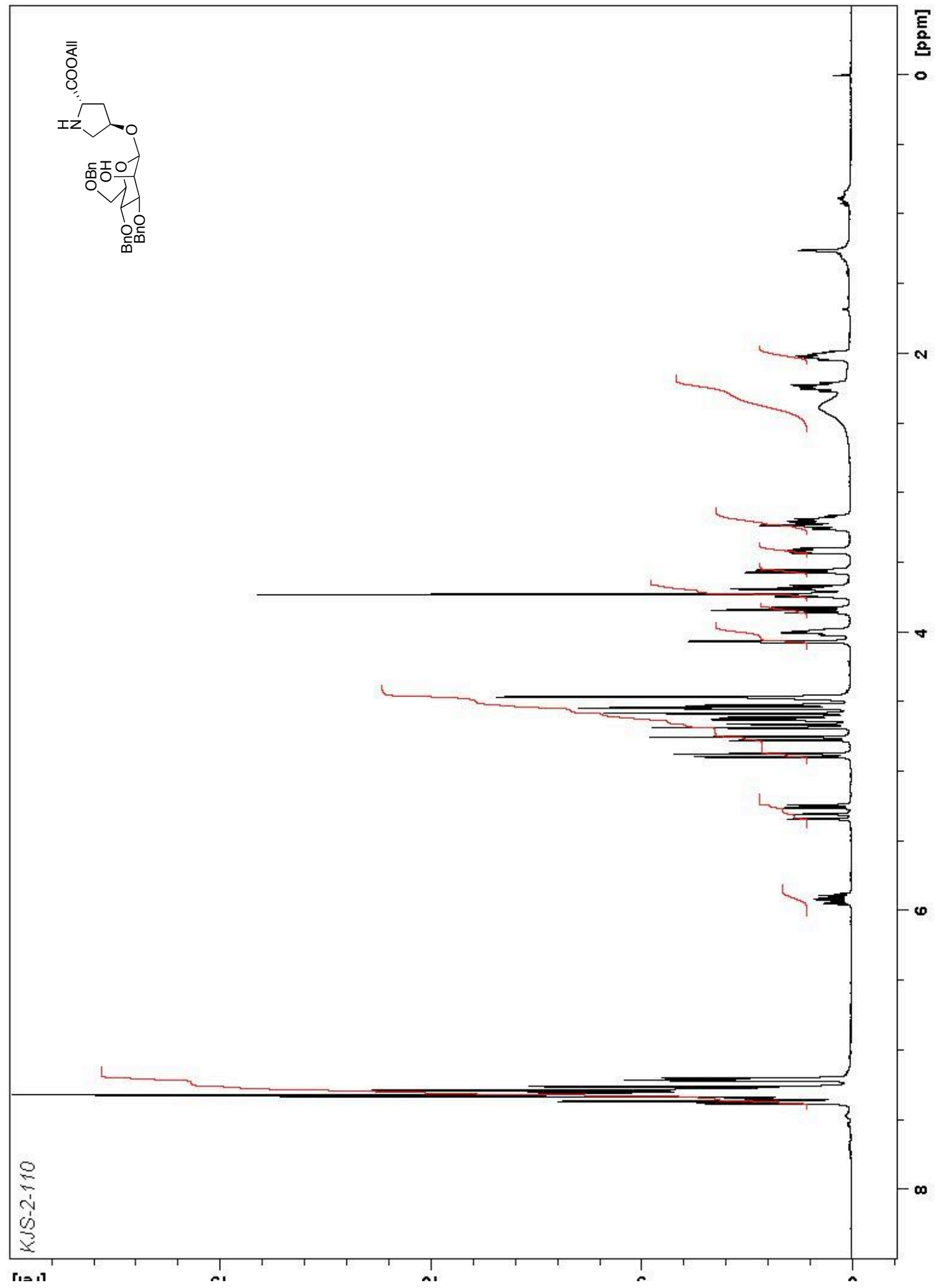


${ }^{13} \mathrm{C}$ NMR spectrum of 4-O-(3,4,6-tri-O-benzyl- $\beta$ - $D$-mannopyranosyl)-trans-4-hydroxy- $L$-proline allyl ester (trans-14), $\mathrm{CDCl}_{3}, 125 \mathrm{MHz}$

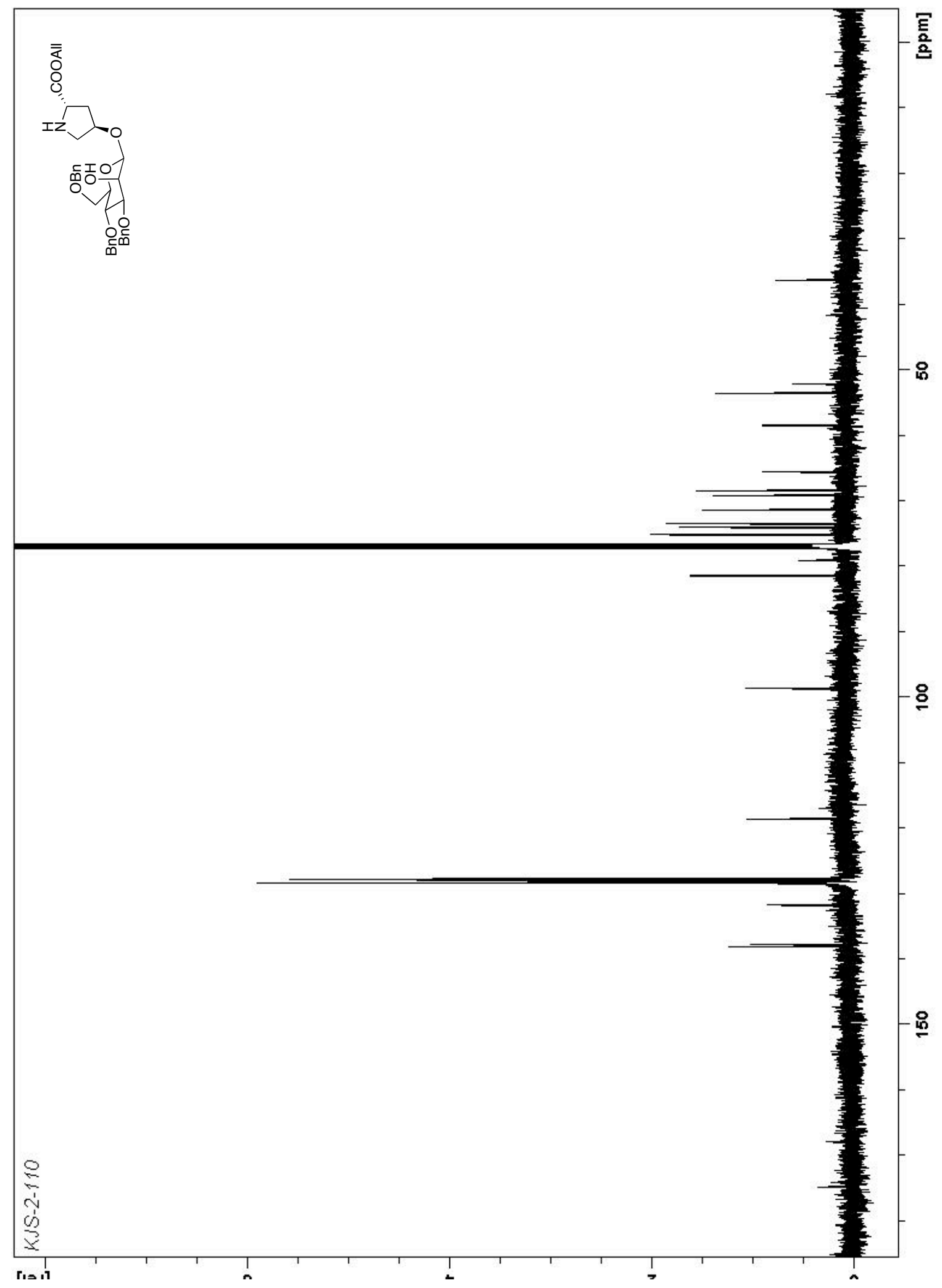


${ }^{1} \mathrm{H}$ NMR spectrum of 4-O-(3,4,6-tri-O-benzyl- $\beta$ - $D$-mannopyranosyl)-cis-4-hydroxy-L-proline allyl ester (cis-14), $\mathrm{CDCl}_{3}, 500 \mathrm{MHz}$

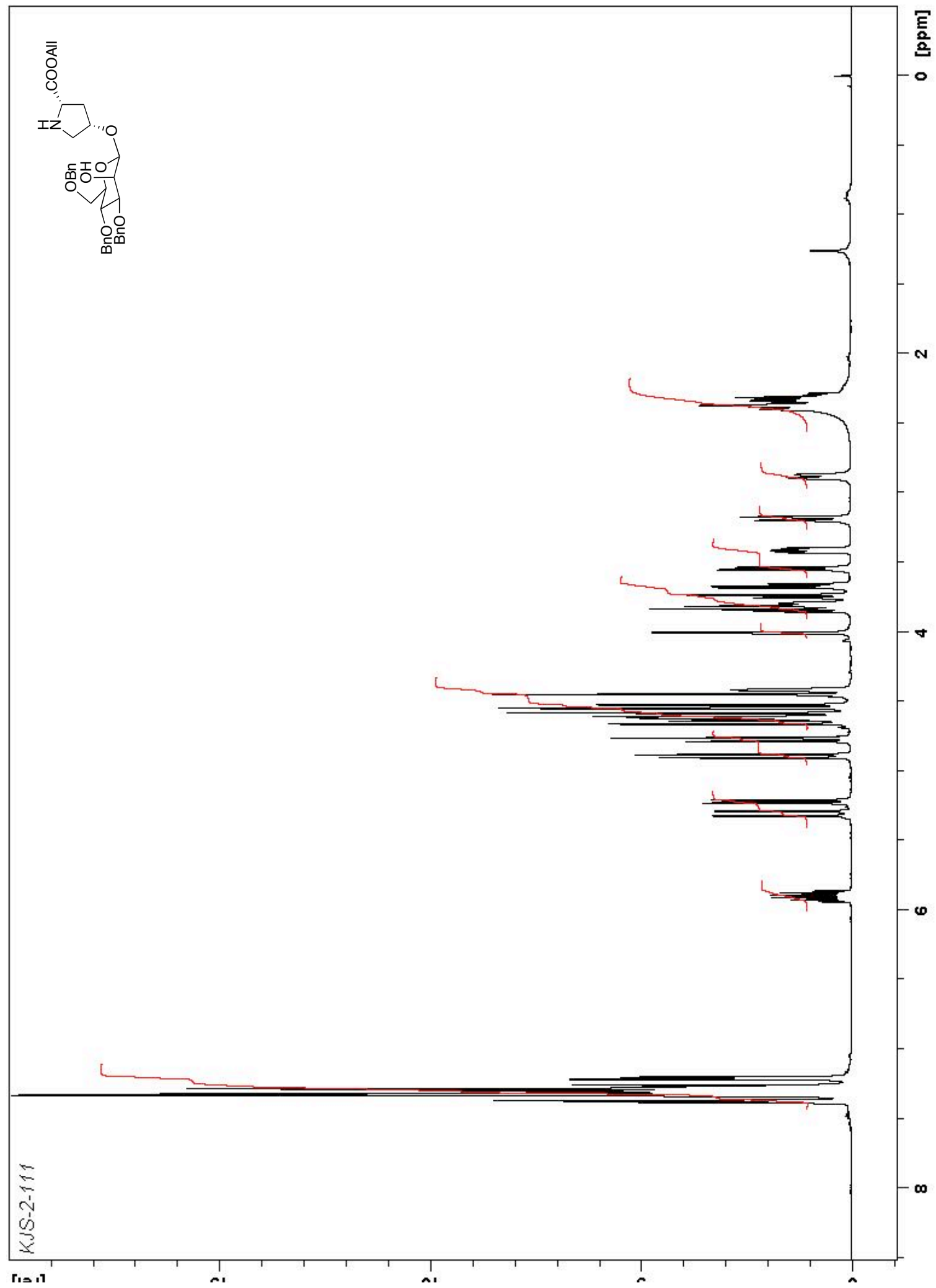


${ }^{13} \mathrm{C}$ NMR spectrum of 4-O-(3,4,6-tri-O-benzyl- $\beta$ - $D$-mannopyranosyl)-cis-4-hydroxy-L-proline allyl ester (cis-14), $\mathrm{CDCl}_{3}, 125 \mathrm{MHz}$

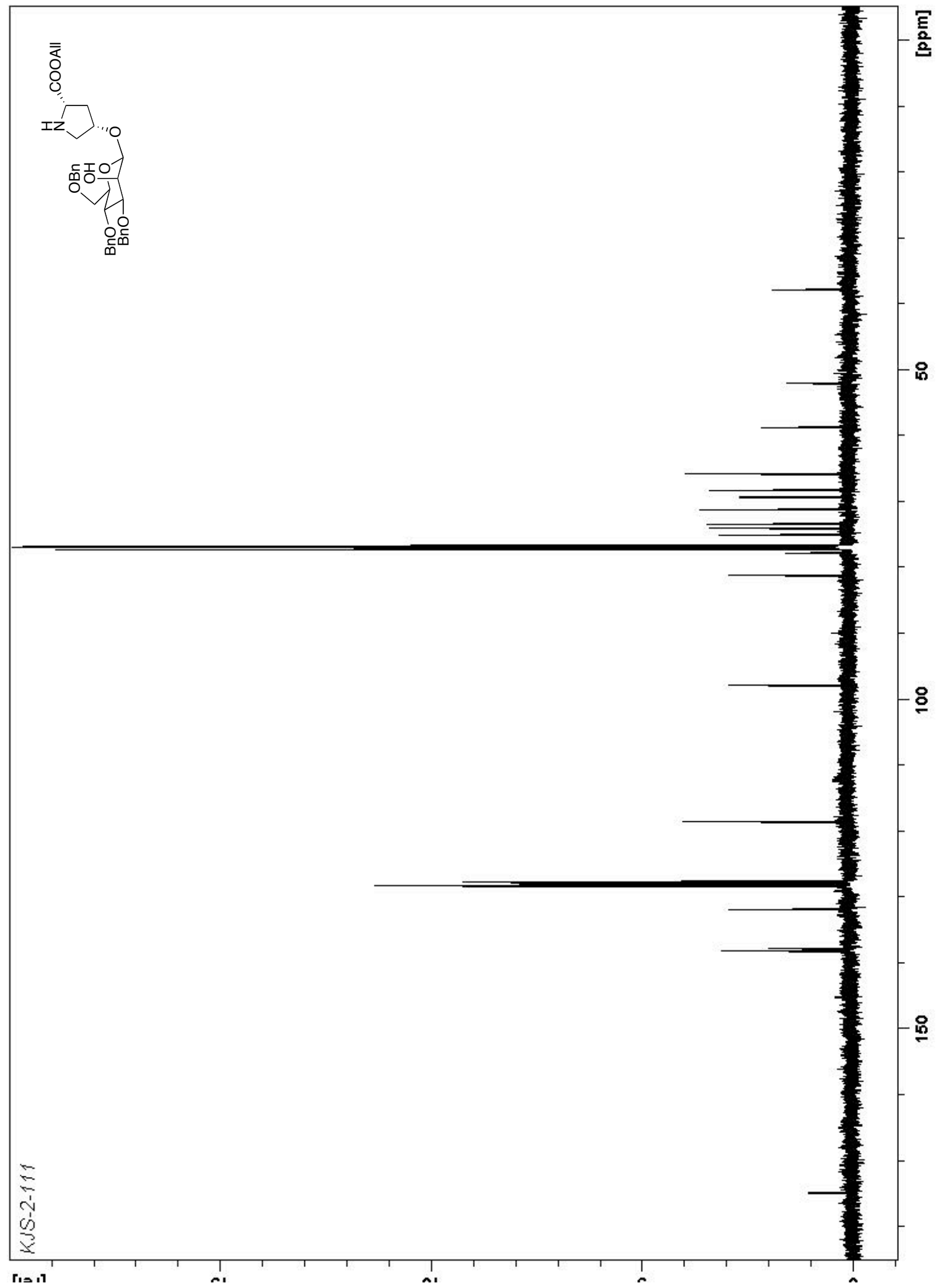


${ }^{1} \mathrm{H}$ NMR spectrum of 4-O-(3,4,6-tri-O-benzyl-O-2-trifluoromethanesulfonyloxy- $\beta$ - $D$-manno-pyranosyl)$N \alpha$-fluorenylmethoxycarbonyl-trans-4-hydroxy- $L$-proline allyl ester (15), $\mathrm{CDCl}_{3}, 500 \mathrm{MHz}$

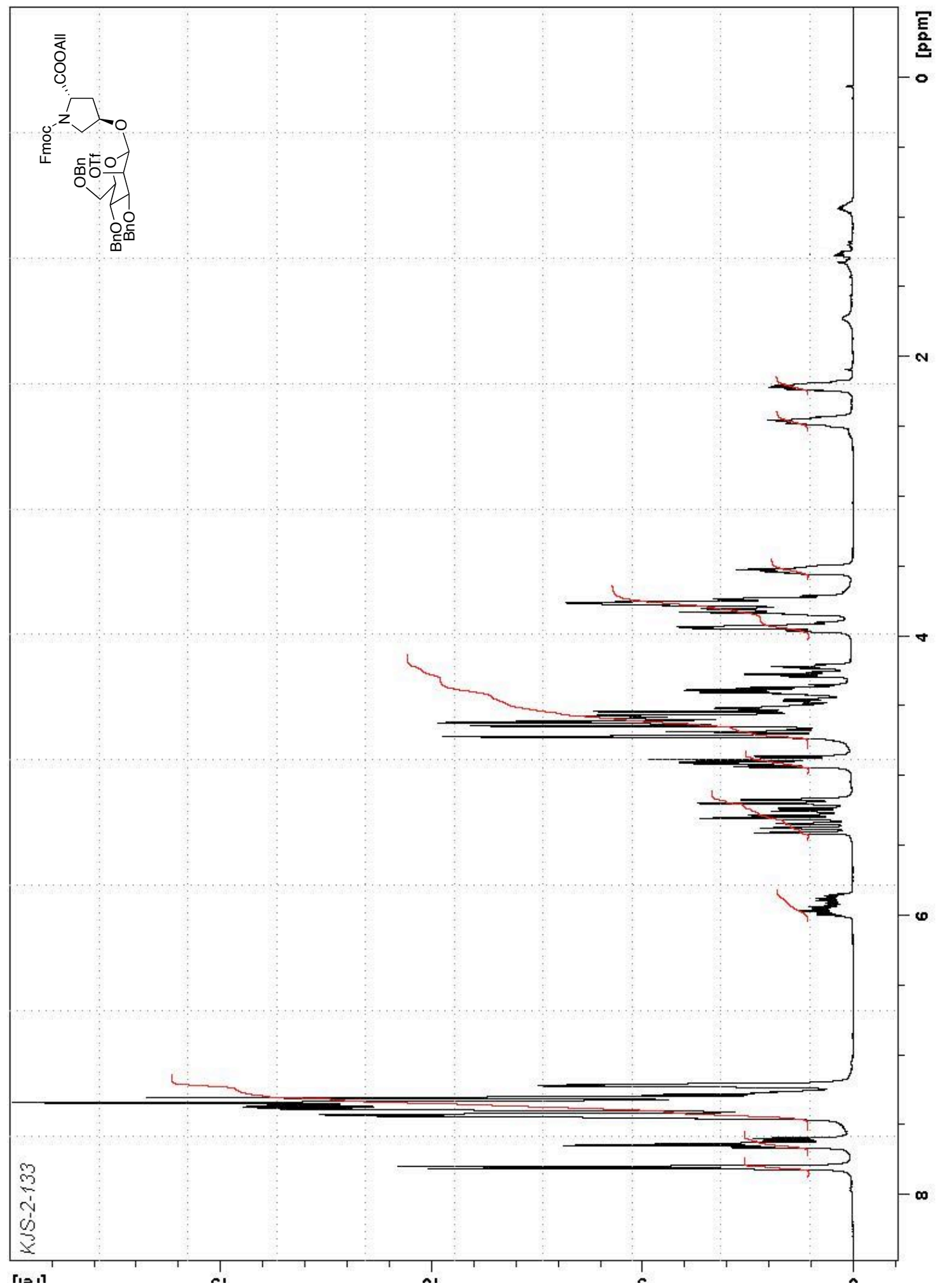


${ }^{13} \mathrm{C}$ NMR spectrum of 4-O-(3,4,6-tri- $O$-benzyl-O-2-trifluoromethanesulfonyloxy- $\beta$ - $D$-mannopyranosyl)$N \alpha$-fluorenylmethoxycarbonyl-trans-4-hydroxy-L-proline allyl ester (15), $\mathrm{CDCl}_{3}, 125 \mathrm{MHz}$

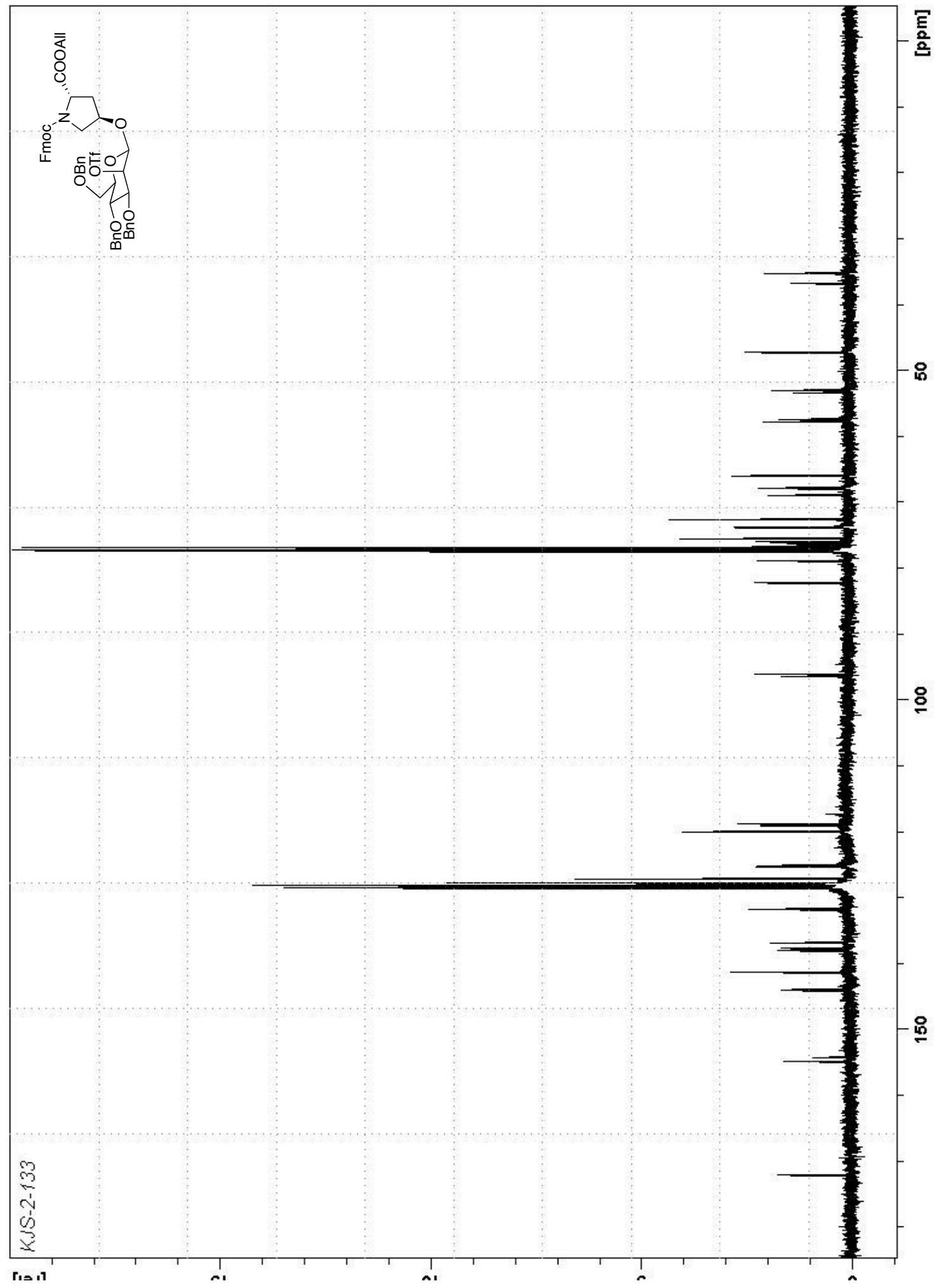


${ }^{1} \mathrm{H}$ NMR spectrum of 4-O-(2-azido-3,4,6-tri- $O$-benzyl-2-deoxy- $\beta$ - $D$-glucopyranosyl)- $N \alpha$ fluorenylmethoxycarbonyl-trans-4-hydroxy-L-proline allyl ester (16), $\mathrm{CDCl}_{3}, 500 \mathrm{MHz}$

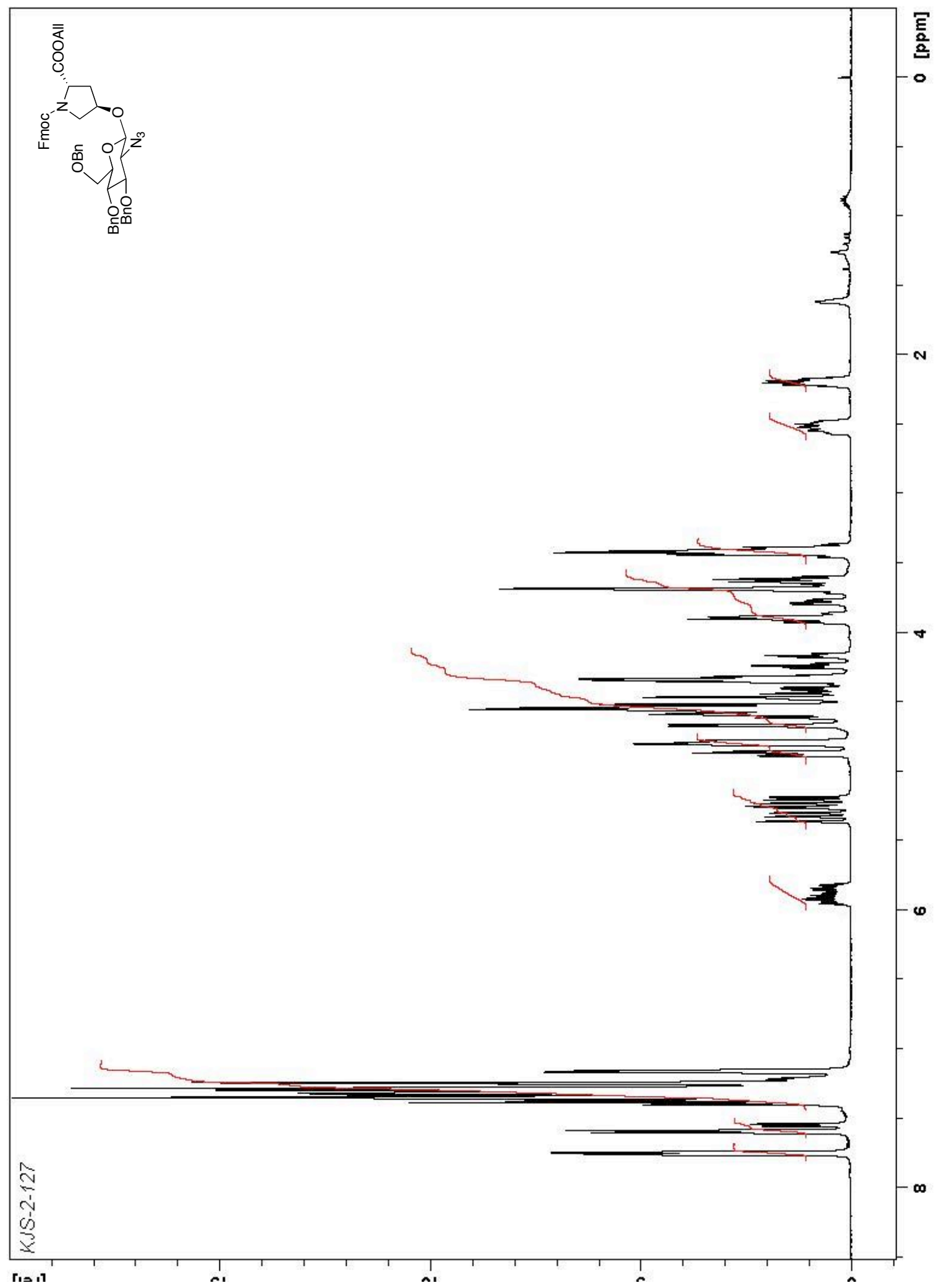


${ }^{13} \mathrm{C}$ NMR spectrum of 4-O-(2-azido-3,4,6-tri-O-benzyl-2-deoxy- $\beta$ - $D$-glucopyranosyl)- $N \alpha$ fluorenylmethoxycarbonyl-trans-4-hydroxy-L-proline allyl ester (16), $\mathrm{CDCl}_{3}, 125 \mathrm{MHz}$

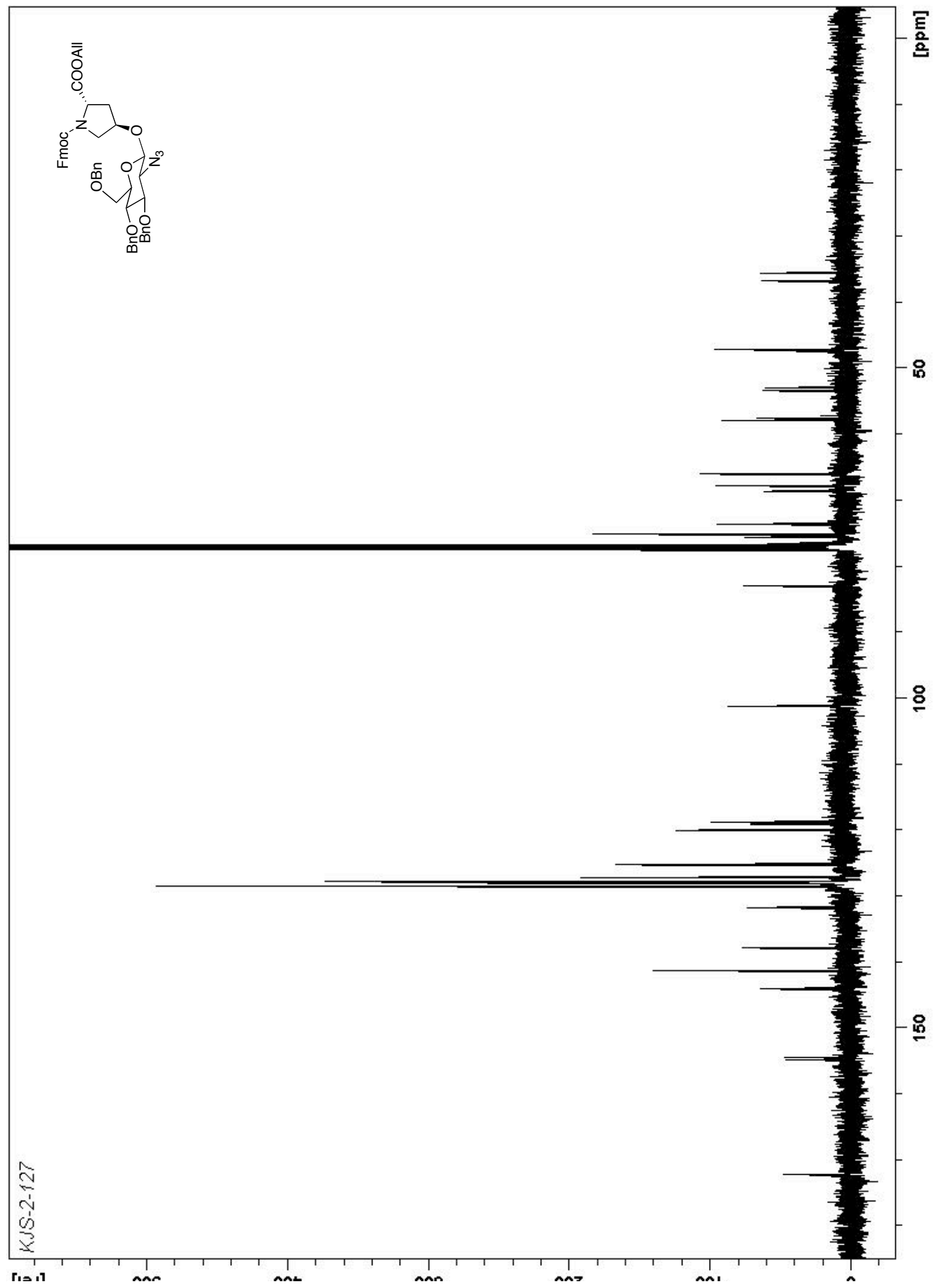


${ }^{1} \mathrm{H}$ NMR spectrum of 4-O-(2-acetamido-3,4,6-tri- $O$-benzyl-2-deoxy- $\beta$ - $D$-glucopyranosyl)- $N \alpha$ fluorenylmethoxycarbonyl-trans-4-hydroxy-L-proline allyl ester (17), $\mathrm{CDCl}_{3}, 500 \mathrm{MHz}$

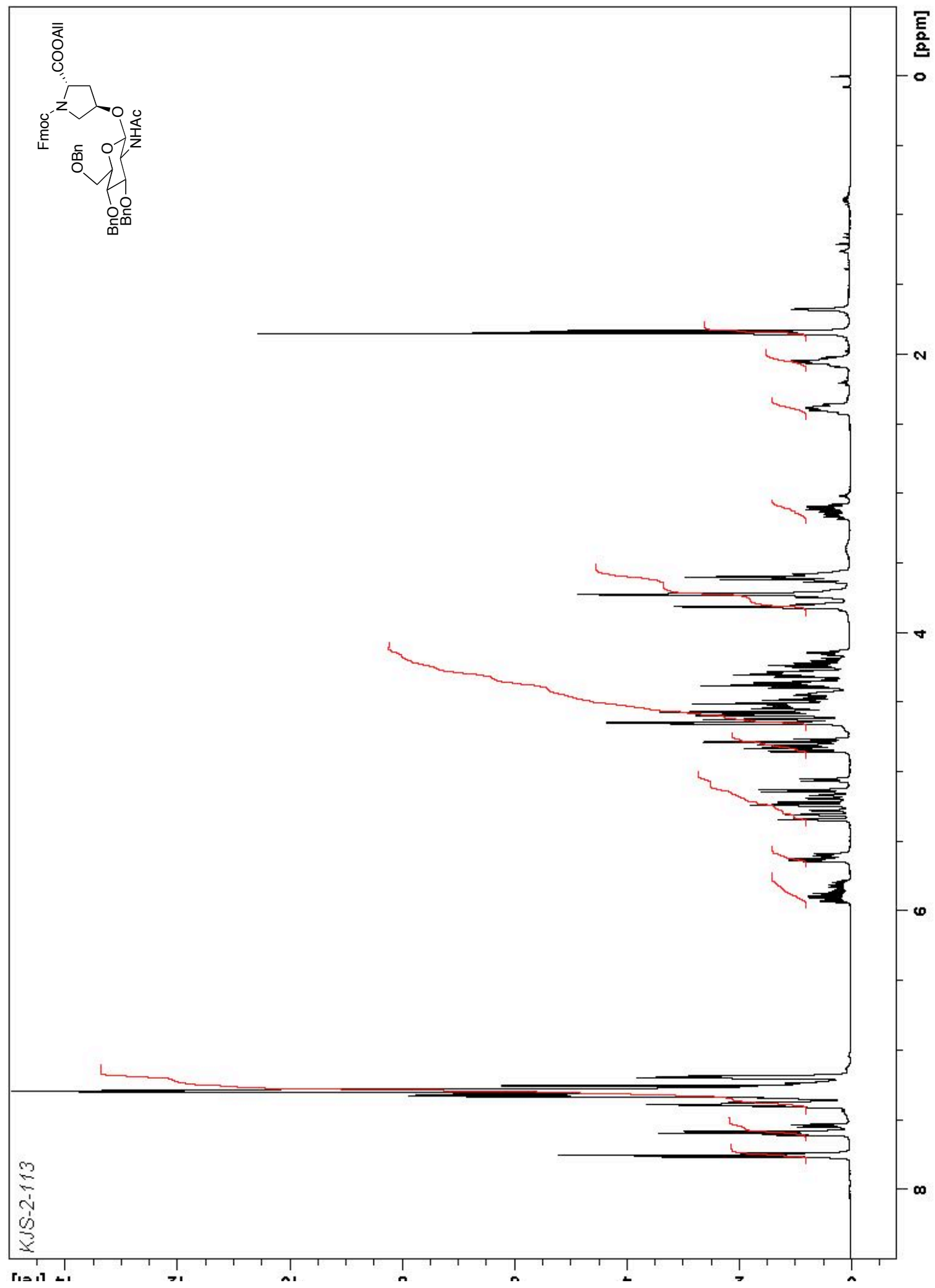


${ }^{13} \mathrm{C}$ NMR spectrum of 4-O-(2-acetamido-3,4,6-tri- $O$-benzyl-2-deoxy- $\beta$ - $D$-glucopyranosyl)- $N \alpha$ fluorenylmethoxycarbonyl-trans-4-hydroxy-L-proline allyl ester (17), $\mathrm{CDCl}_{3}, 125 \mathrm{MHz}$

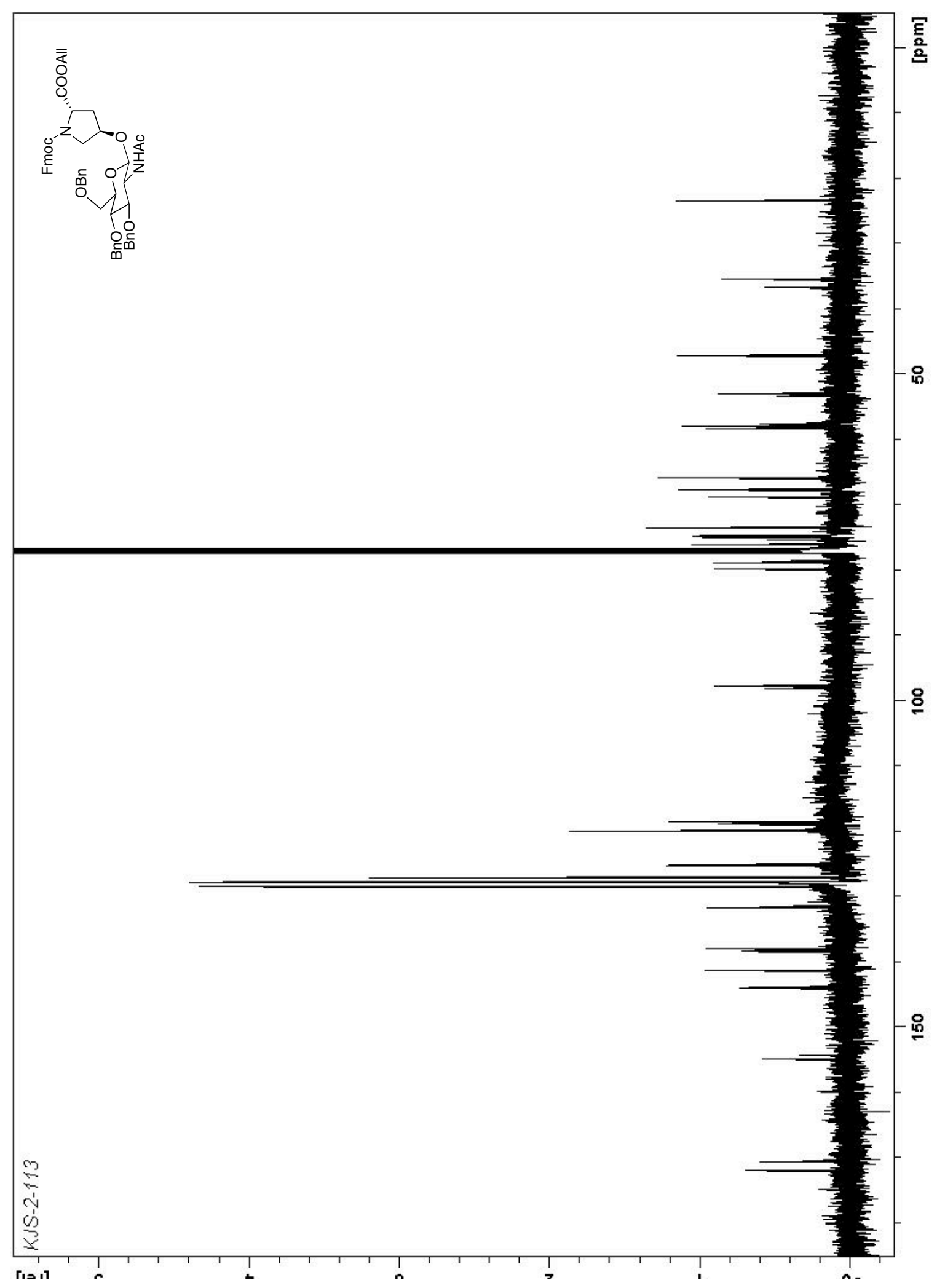


${ }^{1} \mathrm{H}$ NMR spectrum of 4-O-(2-O-benzoyloxy-3,4,6-tri-O-benzyl- $\beta$ - $D$-glucopyranosyl)- $N \alpha$ fluorenylmethoxycarbonyl-trans-4-hydroxy-L-proline allyl ester (18), $\mathrm{CDCl}_{3}, 500 \mathrm{MHz}$

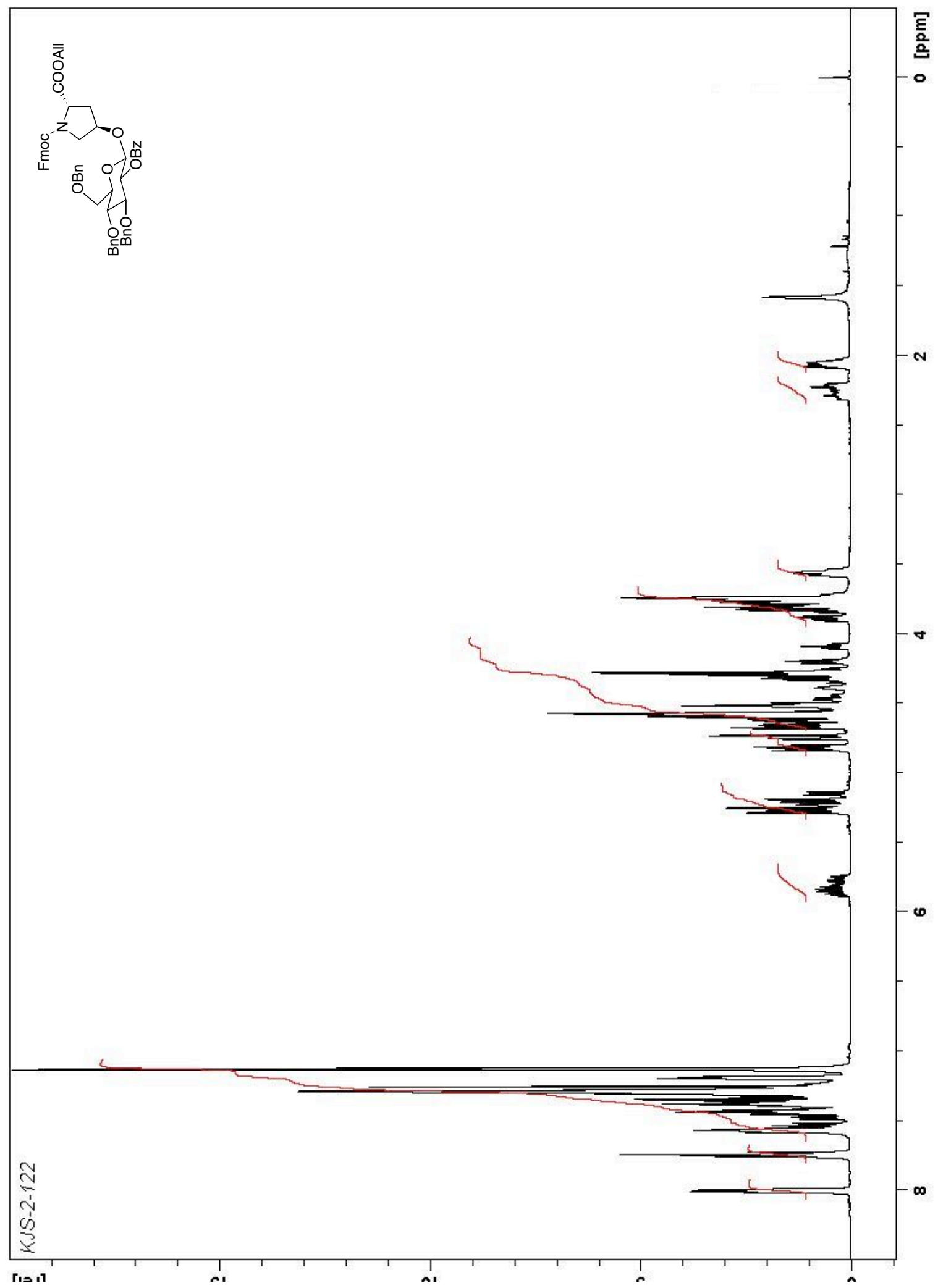


S40

${ }^{13} \mathrm{C}$ NMR spectrum of 4-O-(2-O-benzoyloxy-3,4,6-tri- $O$-benzyl- $\beta$ - $D$-glucopyranosyl)- $N \alpha$ fluorenylmethoxycarbonyl-trans-4-hydroxy-L-proline allyl ester (18), $\mathrm{CDCl}_{3}, 125 \mathrm{MHz}$

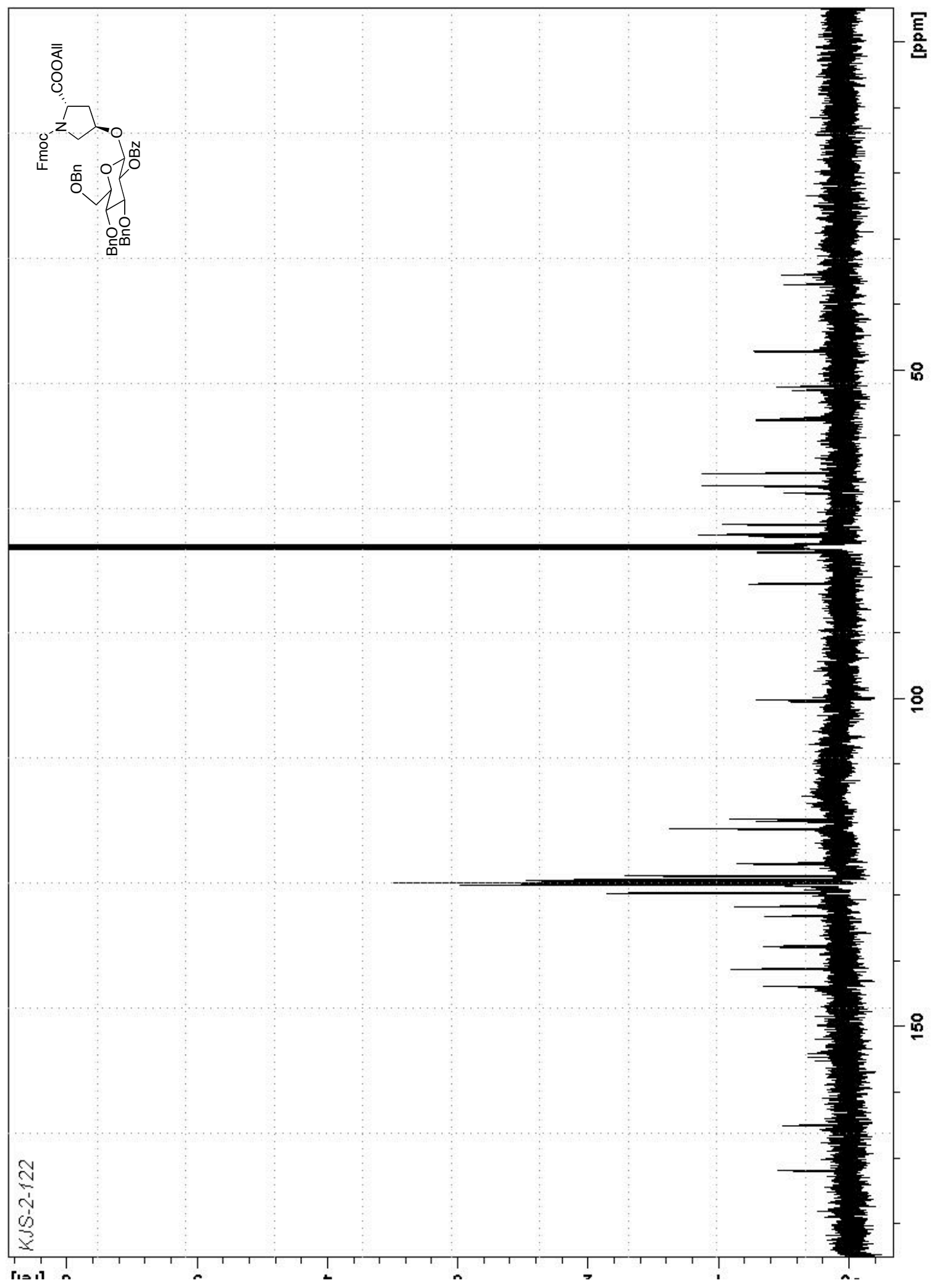

